TITLE:

\title{
Theory of solutions in the energy representation. III. Treatment of the molecular flexibility
}

$\operatorname{AUTHOR}(S)$ :

Matubayasi, N; Nakahara, M

\section{CITATION:}

Matubayasi, N ... [et al]. Theory of solutions in the energy representation. III. Treatment of the molecular flexibility. JOURNAL OF CHEMICAL PHYSICS 2003, 119(18): 9686-9702

\section{ISSUE DATE:}

2003-11-08

URL:

http://hdl.handle.net/2433/50354

\section{RIGHT:}

Copyright 2003 American Institute of Physics. This article may be downloaded for personal use only. Any other use requires prior permission of the author and the American Institute of Physics. 


\title{
Theory of solutions in the energy representation. III. Treatment of the molecular flexibility
}

\author{
Nobuyuki Matubayasi ${ }^{\mathrm{a})}$ and Masaru Nakahara \\ Institute for Chemical Research, Kyoto University, Uji, Kyoto 611-0011, Japan
}

(Received 8 July 2003; accepted 5 August 2003)

\begin{abstract}
The method of energy representation for evaluating the solvation free energy is extended to a solute molecule with structural flexibility. When the intramolecular structure of the solute molecule exhibits a strong response to the solute-solvent interaction, the approximate functional for the solvation free energy needs to be modified from the original form presented previously [J. Chem. Phys. 117, 3605 (2002); 118, 2446 (2003)]. In the modification of the functional, the solvation-induced change in the distribution function of the solute structure is taken into account with respect to the intramolecular energy of the solute. It is then demonstrated over a wide range of thermodynamic conditions that the modified form of functional provides an accurate and efficient route to the solvation free energy of a flexible solute molecule even when the structural distribution function of the solute in solution overlaps barely with that of the solute at isolation. (C) 2003 American Institute of Physics. [DOI: 10.1063/1.1613938]
\end{abstract}

\section{INTRODUCTION}

In a molecular treatment of solutions, the structural flexibility of a molecule is usually excluded from explicit consideration. This is justified for small molecules since the vibrational modes are of high frequency and do not influence intermolecular correlations. The flexibility of a molecule in solution is important when the molecule is large and its intramolecular motion can be coupled to intermolecular interactions. Especially, biomolecules and polymers often involve soft modes in their intramolecular degrees of freedom and may exhibit strong conformational responses to the surrounding environments. Toward understanding and controlling the structure and function of a nanoscale molecule in solution, therefore, it is necessary to establish a statistical mechanical framework to treat the intramolecular flexibility of the molecular structure.

The key quantity to determine the intramolecular structure of a flexible solute molecule in solution is the solvation free energy. Indeed, once the free energy of solvation is evaluated at each fixed structure, the distribution function can be readily obtained for the structure of the solute molecule. ${ }^{1-3}$ The full account of all the intramolecular coordinates is difficult and even unnecessary, however, unless their dimension is low (typically one). A coarse-grained representation of the structural distribution function needs to be introduced for conceptually transparent and computationally convenient description. In a coarse-grained parametrization of the solute structure, a set of solute intramolecular coordinates are chosen as the variables of physical interest and the other coordinates are not of explicit interest. The solvation free energy is then to be expressed in a reduced form over the solute coordinates of interest, and is obtained formally through the integration over the coordinates of no direct interest. The difficulty in practice is that the integration is pos-

a) Author to whom correspondence should be addressed. sible only over a low-dimensional set of coordinates. When the solute degrees of freedom of no interest are multidimensional, they are undesirable to be treated explicitly in the construction of the solvation free energy. Therefore, a scheme is desirably developed which evaluates the solvation free energy of a flexible solute molecule without multidimensional treatment of the intramolecular fluctuations.

In this paper, we present an approach to the solvation free energy of a flexible solute molecule. We adopt the energy representation formulated in previous papers, ${ }^{4-6}$ and provide a modified form of functional for the solvation free energy. In our approach, a one-dimensional coordinate is introduced to describe the intramolecular state of the flexible solute molecule, and the other coordinates are made implicit in the energy distribution functions constituting the functional. It is not required to explicitly list and integrate the free energy of solvation over the multidimensional coordinates for the solute structure. The explicit treatment of the intramolecular degree of freedom is necessary only over a one-dimensional coordinate. A practical approach is then employed, as done in Ref. 5, that computer simulations of the solution and pure solvent systems of interest are performed to obtain the distribution functions constituting the functional for the solvation free energy. This approach to the solvation free energy utilizes the exact solution structure, and its performance is dominated by the degree of approximation involved in the functional.

The purpose of the previous papers was to develop the method of energy representation for evaluating the solvation free energy of a solute molecule in solution. ${ }^{4-6}$ In the energy representation, the coordinate of a solvent molecule around the solute molecule is the solute-solvent interaction energy and the solvent distribution around the solute is expressed over the one-dimensional coordinate for any type of solutesolvent interaction potential. A functional for the solvation free energy was then constructed in terms of energy distribu- 
tion functions of the solution and pure solvent systems of interest, and its performance was demonstrated for nonpolar, polar, and ionic solutes in water over a wide range of thermodynamic conditions including both ambient and supercritical. Actually, the method presented in Refs. 4 and 5 is applicable without modification to a solution of flexible molecules. The energy distribution functions constituting the functional for the solvation free energy can be defined without referring to whether the molecules are rigid or flexible; see Appendix A of Ref. 4. When the distribution function for the solute intramolecular degrees of freedom changes significantly through the interaction with the solvent, however, the solute-solvent interaction potential at the typical structure of the solute is different between the solution and pure solvent systems. In this case, an unmodified application of the approximate method in Refs. 4 and 5 may lead to deterioration of the performance. The developments in the present paper are made to take into account a significant change in the structural distribution of the solute. We extend the approach in Refs. 4 and 5 and demonstrate the performance for simple model systems over a wide range of thermodynamic conditions.

When a set of potential functions is given for the solution system of interest, the "exact" solvation free energy of the solute molecule can be calculated by the free energy perturbation and thermodynamic integration methods. ${ }^{1,7}$ These methods are difficult for a solute molecule with intramolecular degrees of freedom, however, because the coordinate space of the solute needs to be sampled sufficiently at each intermediate state of the gradual process of solute insertion. In contrast, the method developed in this paper and Ref. 5 requires that the computer simulation be performed only at the initial and final states of the solute insertion process. Our method is thus advantageous, especially for a large and/or flexible solute molecule, since the solute is typically involved at dilute condition. The drawback is, of course, that the solvation free energy is evaluated from an approximate functional.

The organization of the present paper is as follows: In Sec. II, a set of distribution functions are introduced to formulate a reduced description of the solute intramolecular state and the solvent configuration relative to the solute molecule, and an approximate functional is constructed for the solvation free energy. In Sec. III, the systems to be examined are identified and the computational procedures are described. In Sec. IV, the performance of the functional for the solvation free energy is assessed in connection with the modification of the solute intramolecular structure due to the solute-solvent interaction.

\section{THEORY}

The system of our interest is a dilute solution containing a single solute molecule. The intermolecular interaction is supposed to be pairwise additive. ${ }^{8}$ The notations and developments in this paper are then parallel to those adopted in Refs. 4 and 5. The complete set of the position and orientation of a solvent molecule is called the full coordinate and is denoted collectively by $\mathbf{x}$. In the present work, the solute molecule involves structural flexibility and its intramolecular degrees of freedom are collectively written as $\psi{ }^{9}$ The solute-solvent interaction potential of interest is $v$ and is fixed at the outset in our developments. Of course, $v$ is a function of $\psi$ and $\mathbf{x}$. It may be expressed as $v^{f}(\psi, \mathbf{x})$ in the full coordinate representation, where a superscript $f$ is attached to emphasize that a function is represented over the full coordinate.

The solvation free energy $\Delta \mu$ is the free energy change corresponding to the gradual insertion process of the solute molecule. ${ }^{6}$ In $\Delta \mu$, only the contribution from the potential energy is involved and the ideal (kinetic) contribution is excluded. When the intramolecular energy of the solute is $\Psi(\psi)$ and the solvent-solvent interaction energy is $U(\mathbf{X}), \Delta \mu$ is expressed as

$$
\begin{aligned}
\exp (-\beta \Delta \mu) & \\
= & \frac{\int d \psi d \mathbf{X} \exp \left(-\beta\left\{\Psi(\psi)+\Sigma_{i} v^{f}\left(\psi, \mathbf{x}_{i}\right)+U(\mathbf{X})\right\}\right)}{\int d \psi d \mathbf{X} \exp (-\beta\{\Psi(\psi)+U(\mathbf{X})\})},
\end{aligned}
$$

where $\mathbf{X}$ represents the solvent configuration collectively and $\mathbf{x}_{i}$ is the full coordinate of the $i$ th solvent molecule. $\beta$ is the inverse of $k_{B} T$, as in the usual notational convention, with the Boltzmann constant $k_{B}$ and the temperature $T$. A restriction of attention to a certain set of solute intramolecular state can be made simply by the corresponding alteration of the domain of integration over $\psi$. Especially, when the solute structure is fixed at a specific $\psi$, the solvation free energy $\Delta \sigma(\psi)$ at that $\psi$ is written as

$\exp (-\beta \Delta \sigma(\psi))=\frac{\int d \mathbf{X} \exp \left(-\beta\left\{\Sigma_{i} v^{f}\left(\psi, \mathbf{x}_{i}\right)+U(\mathbf{X})\right\}\right)}{\int d \mathbf{X} \exp (-\beta U(\mathbf{X}))}$.

$\Delta \mu$ is then related to $\Delta \sigma(\psi)$ through

$$
\exp (-\beta \Delta \mu)=\int d \psi p_{0}(\psi) \exp (-\beta \Delta \sigma(\psi))
$$

where $p_{0}(\psi)$ is the probability distribution function of $\psi$ for the solute at isolation (absence of the solute-solvent interaction) and is given by

$$
p_{0}(\psi)=\frac{\exp (-\beta \Psi(\psi))}{\int d \psi \exp (-\beta \Psi(\psi))} .
$$

It should be noted in Eq. (3) that although $\Delta \mu$ is obtained from an integration of $\Delta \sigma(\psi)$ over the solute intramolecular coordinate $\psi$, it is not an average (weighted sum) of $\Delta \sigma(\psi)$.

The distribution of the solute intramolecular coordinate $\psi$ is modified from Eq. (4) upon introduction of the solutesolvent interaction $v$. In the solution system of interest, the probability distribution function $p(\psi)$ is expressed as

$p(\psi)=\frac{\int d \mathbf{X} \exp \left(-\beta\left\{\Psi(\psi)+\sum_{i} v^{f}\left(\psi, \mathbf{x}_{i}\right)+U(\mathbf{X})\right\}\right)}{\int d \psi d \mathbf{X} \exp \left(-\beta\left\{\Psi(\psi)+\Sigma_{i} v^{f}\left(\psi, \mathbf{x}_{i}\right)+U(\mathbf{X})\right\}\right)}$.

According to Eqs. (1), (2), and (4),

$$
p(\psi)=p_{0}(\psi) \exp (-\beta(\Delta \sigma(\psi)-\Delta \mu))
$$

holds exactly at each value of $\psi \cdot{ }^{10}$ It should be noted that Eq. (6) corresponds in form to Eq. (19) of Ref. 11, which relates the solvation free energy to the total solute-solvent interaction and its distributions in the solution and pure solvent systems of interest. ${ }^{11,12}$ 
The energy representation is introduced by adopting the value of the solute-solvent interaction $v$ of interest as the coordinate $\epsilon$ for the distribution of the solvent molecule around the solute molecule. The instantaneous distribution $\hat{\rho}^{e}$ is then defined as

$$
\hat{\rho}^{e}(\boldsymbol{\epsilon})=\sum_{i} \delta\left(v^{f}\left(\psi, \mathbf{x}_{i}\right)-\boldsymbol{\epsilon}\right),
$$

where the sum is taken over all the solvent molecules and a superscript $e$ is attached to emphasize that a function is represented over the energy coordinate. This definition is of course parallel to that given in Refs. 4 and 5. Note that the specification of $v$ is necessary in Eq. (7).

In Ref. 5, the solvation free energy $\Delta \mu$ is expressed in terms of distribution functions constructed from $\hat{\rho}^{e}$ in the solution and pure solvent systems. In our treatments, the solution system refers to the system in which the solute molecule interacts with the solvent under the solute-solvent interaction $v$ of interest at full coupling. In the solution, the average distribution $\rho^{e}$ of the $v$ value is given by

$$
\rho^{e}(\epsilon)=\left\langle\hat{\rho}^{e}(\epsilon)\right\rangle_{v}
$$

where $\langle Q\rangle_{v}$ represents the ensemble average of a quantity $Q$ and is written as

$$
\begin{aligned}
\langle Q\rangle_{v} & =\frac{\int d \psi d \mathbf{X} Q \exp \left(-\beta\left\{\Psi(\psi)+\Sigma_{i} v^{f}\left(\psi, \mathbf{x}_{i}\right)+U(\mathbf{X})\right\}\right)}{\int d \psi d \mathbf{X} \exp \left(-\beta\left\{\Psi(\psi)+\Sigma_{i} v^{f}\left(\psi, \mathbf{x}_{i}\right)+U(\mathbf{X})\right\}\right)} \\
& =\int d \psi p(\psi) \frac{\int d \mathbf{X} Q \exp \left(-\beta\left\{\Sigma_{i} v^{f}\left(\psi, \mathbf{x}_{i}\right)+U(\mathbf{X})\right\}\right)}{\int d \mathbf{X} \exp \left(-\beta\left\{\Sigma_{i} v^{f}\left(\psi, \mathbf{x}_{i}\right)+U(\mathbf{X})\right\}\right)}
\end{aligned}
$$

in terms of the distribution function $p(\psi)$ of the solute intramolecular coordinate $\psi$ in the solution system. On the other hand, the pure solvent system denotes the system in which no interaction is physically present between the solute and solvent molecules. At an instantaneous configuration of the pure solvent system, $\hat{\rho}^{e}$ is constructed by placing the solute molecule in the neat solvent system as a test particle. The average distribution $\rho_{0}^{e}$ and the correlation matrix $\chi_{0}^{e}$ are then expressed, respectively, as

$$
\rho_{0}^{e}(\epsilon)=\left\langle\hat{\rho}^{e}(\epsilon)\right\rangle_{0}
$$

and

$$
\chi_{0}^{e}(\epsilon, \eta)=\left\langle\hat{\rho}^{e}(\epsilon) \hat{\rho}^{e}(\eta)\right\rangle_{0}-\left\langle\hat{\rho}^{e}(\epsilon)\right\rangle_{0}\left\langle\hat{\rho}^{e}(\eta)\right\rangle_{0},
$$

where $\langle Q\rangle_{0}$ is the ensemble average and is given by

$$
\begin{aligned}
\langle Q\rangle_{0} & =\frac{\int d \psi d \mathbf{X} Q \exp (-\beta\{\Psi(\psi)+U(\mathbf{X})\})}{\int d \psi d \mathbf{X} \exp (-\beta\{\Psi(\psi)+U(\mathbf{X})\})} \\
& =\int d \psi p_{0}(\psi) \frac{\int d \mathbf{X} Q \exp (-\beta U(\mathbf{X}))}{\int d \mathbf{X} \exp (-\beta U(\mathbf{X}))}
\end{aligned}
$$

with the distribution $p_{0}(\psi)$ of the solute intramolecular coordinate $\psi$ in the absence of the solute-solvent interaction. It should be noted in Eq. (12) that the solute and solvent degrees of freedom are uncoupled from each other in the probability distribution. This equation shows that the solute molecule is placed as a test particle in the neat solvent system.

By following the procedures in Ref. 5, it is straightforward to construct a functional for the solvation free energy $\Delta \mu$ in terms of the energy distribution functions $\rho^{e}, \rho_{0}^{e}$, and $\chi_{0}^{e} \cdot{ }^{6}$ According to the approximation adopted in Ref. $5, \Delta \mu$ is given by a set of equations listed as

$$
\begin{aligned}
w^{e}(\epsilon)= & -k_{B} T \log \left(\frac{\rho^{e}(\epsilon)}{\rho_{0}^{e}(\epsilon)}\right)-\epsilon, \\
w_{0}^{e}(\epsilon)= & -k_{B} T \int d \eta\left(\frac{\delta(\epsilon-\eta)}{\rho_{0}^{e}(\epsilon)}-\left(\chi_{0}^{e}\right)^{-1}(\epsilon, \eta)\right) \\
& \times\left(\rho^{e}(\eta)-\rho_{0}^{e}(\eta)\right), \\
\Delta \mu=- & k_{B} T \int d \epsilon\left[\left(\rho^{e}(\epsilon)-\rho_{0}^{e}(\epsilon)\right)+\beta w^{e}(\epsilon) \rho^{e}(\epsilon)\right. \\
- & \left.\left\{\alpha(\epsilon) F(\epsilon)+(1-\alpha(\epsilon)) F_{0}(\epsilon)\right\}\left(\rho^{e}(\epsilon)-\rho_{0}^{e}(\epsilon)\right)\right],
\end{aligned}
$$


The above set of equations for the solvation free energy $\Delta \mu$ is exactly the same in form as that given in Ref. 5. The only difference is the trivial redefinition of the instantaneous distribution $\hat{\rho}^{e}$ by Eq. (7). In this sense, the method presented in Refs. 4 and 5 is applicable to a flexible solute molecule without modification.

In Eq. (3), the solvation free energy $\Delta \mu$ is expressed as an integral over the solute intramolecular coordinate $\psi$. It is then seen by virtue of Eq. (6) that the main contribution to the integral comes from the region of $\psi$ in which the distribution function $p(\psi)$ in the solution system is large. ${ }^{10}$ When the energy distribution function $\rho^{e}$ is to be obtained, $\psi$ is sampled in the solution system and $p(\psi)$ is realized. To construct $\rho_{0}^{e}$ and $\chi_{0}^{e}$ in the pure solvent system, in contrast, the sampling of $\psi$ is performed according to the distribution function $p_{0}(\psi)$, not to $p(\psi)$. The variation in the structural distribution of the solute is accompanied by the change in the solute-solvent interaction potential at typical values of $\psi$. When $p(\psi)$ overlaps barely with $p_{0}(\psi)$, in particular, the content of the energy coordinate $\epsilon$ is effectively different between the solution and pure solvent systems. In this case, the approximate scheme may not perform well in the original form given by Eqs. (13)-(18), and needs to be modified to take into account the difference between $p(\psi)$ and $p_{0}(\psi)$. Our modification for treating a flexible solute molecule is to devise a reference solute molecule for which the distribution function of the solute intramolecular coordinate $\psi$ is revised from $p_{0}(\psi)$ and reflects some representative features of $p(\psi)$.

In order to introduce the reference solute molecule, we employ a function $\Phi(\psi)$ defined over the solute intramolecular coordinate $\psi . \Phi(\psi)$ characterizes the solute intramolecular state in a conceptually and computationally convenient manner. Of course, $\Phi(\psi)$ can be taken to be $\psi$ itself. This choice is not useful, however, when $\psi$ is multidimensional. It is advantageous in practice to adopt a one-dimensional $\Phi$ by reducing the information content for the solute structure. At the end of this section, $\Phi$ is actually set to the intramolecular potential $\Psi$ of the solute, although the following developments can be made without specifying the explicit form of the function $\Phi$. Since the formulation is common to any form of $\Phi$, we keep the form generic until the end of the section.

The probability distribution function $P(\phi)$ of the value $\phi$ of the function $\Phi(\psi)$ is expressed in the solution system as

$$
P(\phi)=\int d \psi \delta(\phi-\Phi(\psi)) p(\psi)
$$

Similarly, the probability distribution function $P_{0}(\phi)$ for the solute molecule at isolation (absence of the solute-solvent interaction) is given by

$$
P_{0}(\phi)=\int d \psi \delta(\phi-\Phi(\psi)) p_{0}(\psi)
$$

The reference solute molecule is defined in terms of these probability distribution functions. When the solute is subject to the intramolecular potential function $\Psi(\psi)$ in its original form, the reference solute molecule adopts

$$
\Psi(\psi)-k_{B} T \log \left(\frac{P(\Phi(\psi))}{P_{0}(\Phi(\psi))}\right)
$$

as the intramolecular potential. In Eq. (21), the second term incorporates the effect of the solute-solvent interaction on $\Phi(\psi)$ in the form of distribution and gives rise to the difference between the original and reference solute molecules. It should be noted that Eq. (21) is a function of the intramolecular coordinate $\psi$ of the solute. $P$ and $P_{0}$ are constructed from Eqs. (19) and (20), respectively, and their arguments $\Phi(\psi)$ are defined over $\psi$. When Eq. (21) is taken to be the solute intramolecular energy in the absence of solvent, the probability distribution function $\widetilde{p}_{0}(\psi)$ of $\psi$ is expressed as

$$
\widetilde{p}_{0}(\psi)=\frac{P(\Phi(\psi))}{P_{0}(\Phi(\psi))} p_{0}(\psi) .
$$

Equation (22) shows that the conditional probability distribution of $\psi$ at a fixed value $\phi$ of $\Phi(\psi)$ is the same between the original and reference solute molecules and that the $\phi$ distribution for the reference solute molecule is identical to the distribution in the solution system of interest. When the reference solute molecule is placed in the neat solvent system as a test particle, the corresponding ensemble average $\langle Q\rangle_{\Phi}$ of a quantity $Q$ is given by

$$
\begin{aligned}
\langle Q\rangle_{\Phi} & =\int d \psi \widetilde{p}_{0}(\psi) \frac{\int d \mathbf{X} Q \exp (-\beta U(\mathbf{X}))}{\int d \mathbf{X} \exp (-\beta U(\mathbf{X}))} \\
& =\int d \phi P(\phi)\langle Q\rangle_{\phi},
\end{aligned}
$$

where $\langle Q\rangle_{\phi}$ is the conditional average at a fixed $\phi$ and is written as

$$
\langle Q\rangle_{\phi}=\frac{\int d \psi d \mathbf{X} \delta(\phi-\Phi(\psi)) Q \exp (-\beta\{\Psi(\psi)+U(\mathbf{X})\})}{\int d \psi d \mathbf{X} \delta(\phi-\Phi(\psi)) \exp (-\beta\{\Psi(\psi)+U(\mathbf{X})\})} .
$$

$\langle Q\rangle_{\phi}$ is not affected by the replacement of $\Psi(\psi)$ with Eq. (21). In other words, the conditional average at a fixed $\phi$ is invariant when the solute molecule is changed from the original to the reference. The modification of the solute intramolecular state in the solution is reflected only through $P(\phi)$ in Eq. (23). It should be noted furthermore that the solute and solvent degrees of freedom in the probability distribution are apparently uncoupled from each other in Eq. (23).

With its value $\phi$, the function $\Phi(\psi)$ specifies the domain of the solute intramolecular coordinate $\psi$. When a condition $\Phi(\psi)=\phi$ is imposed, the free energy change $\Delta \nu(\phi)$ for the solute insertion is expressed as

$$
\exp (-\beta \Delta \nu(\phi))=\frac{\int d \psi d \mathbf{X} \delta(\phi-\Phi(\psi)) \exp \left(-\beta\left\{\Psi(\psi)+\sum_{i} v^{f}\left(\psi, \mathbf{x}_{i}\right)+U(\mathbf{X})\right\}\right)}{\int d \psi d \mathbf{X} \delta(\phi-\Phi(\psi)) \exp (-\beta\{\Psi(\psi)+U(\mathbf{X})\})}
$$

This equation is similar in form to Eq. (3) and is rewritten as 


$$
\exp (-\beta \Delta \nu(\phi))=\frac{\int d \psi \delta(\phi-\Phi(\psi)) p_{0}(\psi) \exp (-\beta \Delta \sigma(\psi))}{\int d \psi \delta(\phi-\Phi(\psi)) p_{0}(\psi)}
$$

where $\Delta \sigma(\psi)$ is introduced by Eq. (2). According to Eqs. (3) and (26), $\Delta \nu(\phi)$ is the conditional solvation free energy specified by $\Phi(\psi)=\phi$, and is related to the (total) solvation free energy $\Delta \mu$ given by Eq. (1) through

$$
\exp (-\beta \Delta \mu)=\int d \phi P_{0}(\phi) \exp (-\beta \Delta \nu(\phi)) .
$$

The hierarchical structure is evident in Eqs. (3), (26), and (27). The coordinate $\phi$ provides a coarse-grained description of the solute intramolecular state, and $\Delta \nu(\phi)$ is an "intermediate" between $\Delta \sigma(\psi)$ and $\Delta \mu$ in the construction of the solvation free energy.

The probability distribution functions $P(\phi)$ and $P_{0}(\phi)$ are related to each other through an expression similar to Eq. (6). Indeed, it follows from Eqs. (19), (20), and (26) that

$$
P(\phi)=P_{0}(\phi) \exp (-\beta(\Delta \nu(\phi)-\Delta \mu))
$$

holds exactly at each value of $\phi$ for any choice of the function $\Phi(\psi) .{ }^{10}$ Especially, the solvation free energy $\Delta \mu$ is written in terms of its conditional counterpart $\Delta \nu(\phi)$ as

$$
\Delta \mu=\Delta \tilde{\mu}+k_{B} T \int d \phi P(\phi) \log \left(\frac{P(\phi)}{P_{0}(\phi)}\right),
$$

where $\Delta \tilde{\mu}$ is given by

$$
\Delta \widetilde{\mu}=\int d \phi P(\phi) \Delta \nu(\phi) .
$$

Unlike Eqs. (3), (26), and (27), Eq. (30) introduces $\Delta \tilde{\mu}$ as an integral of $\Delta \nu(\phi)$ weighted by $P(\phi) . \Delta \tilde{\mu}$ is simply the average of the component free energy change $\Delta \nu(\phi)$. The second term of Eq. (29) represents the cross entropy of the distribution $P(\phi)$ relative to $P_{0}(\phi)$ in the unit of $-k_{B} T$. This term is always non-negative, ${ }^{13}$ so that

$$
\Delta \mu \geqslant \Delta \tilde{\mu}
$$

is an exact inequality. Equation (29) is actually of the form of free energy when $\Delta \nu(\phi)$ is considered an effective "energy" at the state specified by $\phi$. The first term is the average "energy" and the second term makes the entropic contribution. ${ }^{14}$

An average of the free energy (change) of the form Eq. 30 appears commonly in the theoretical treatment of the systems with quenched degrees of freedom, such as spin glass and fluid in confined medium. ${ }^{15-20}$ The standard technique for studying this type of average is the replica method ${ }^{15}$ and is recently extended to fluid system. ${ }^{17-20}$ In the present work, we formulate an approximate expression for $\Delta \tilde{\mu}$ on the basis of the density-functional theory in the energy representation established in Ref. 4. When the energy coordinate $\epsilon$ is introduced with respect to the solute-solvent interaction $v$ of interest, the correspondence is one-to-one in the energy representation from the solute-solvent interaction potential to the solvent distribution around the solute. In Appendix A, it is proven that the one-to-one correspondence is also valid for a set of systems with an identical probability distribution $P(\phi)$ of the value $\phi$ of the function $\Phi(\psi)$. An approximation to $\Delta \tilde{\mu}$ can then be formulated in parallel with that presented in Ref. 5. The detail of the formulation is described in Appendix A, and only the final expression is shown in this section.

When the solvation free energy $\Delta \mu$ is to be evaluated through the original form of approximation listed as Eqs. (13) - (18), the inputs needed are the energy distribution functions $\rho^{e}, \rho_{0}^{e}$, and $\chi_{0}^{e}$ given by Eqs. (8), (10), and (11), respectively. The approximation to $\Delta \tilde{\mu}$ is expressed, on the other hand, in terms of $\rho^{e}$ in the solution system of interest and two distribution functions $\widetilde{\rho}_{0}^{e}$ and $\widetilde{\chi}_{0}^{e}$ in the pure solvent system which involves the reference solute molecule as a test particle. $\widetilde{\rho}_{0}^{e}$ is the average of the instantaneous distribution $\hat{\rho}^{e}(\epsilon)$ defined by Eq. (7) and is written as

$$
\widetilde{\rho}_{0}^{e}(\epsilon)=\left\langle\hat{\rho}^{e}(\epsilon)\right\rangle_{\Phi}=\int d \phi P(\phi)\left\langle\hat{\rho}^{e}(\epsilon)\right\rangle_{\phi},
$$

where $P(\phi)$ is the probability distribution function in the solution system given by Eq. (19) and $\langle\cdots\rangle_{\Phi}$ and $\langle\cdots\rangle_{\phi}$ are the averages introduced by Eqs. (23) and (24), respectively. $\widetilde{\chi}_{0}^{e}$ is the correlation matrix set to

$$
\begin{aligned}
\widetilde{\chi}_{0}^{e}(\epsilon, \eta)= & \left\langle\hat{\rho}^{e}(\epsilon) \hat{\rho}^{e}(\eta)\right\rangle_{\Phi} \\
& -\int d \phi P(\phi)\left\langle\hat{\rho}^{e}(\epsilon)\right\rangle_{\phi}\left\langle\hat{\rho}^{e}(\eta)\right\rangle_{\phi} \\
= & \int d \phi P(\phi)\left(\left\langle\hat{\rho}^{e}(\epsilon) \hat{\rho}^{e}(\eta)\right\rangle_{\phi}\right. \\
& \left.-\left\langle\hat{\rho}^{e}(\epsilon)\right\rangle_{\phi}\left\langle\hat{\rho}^{e}(\eta)\right\rangle_{\phi}\right) .
\end{aligned}
$$

It is actually different from the second cumulant of $\hat{\rho}^{e}(\epsilon)$ in the pure solvent system with the reference solute molecule by

$$
\begin{aligned}
& \int d \phi P(\phi)\left\langle\hat{\rho}^{e}(\epsilon)\right\rangle_{\phi}\left\langle\hat{\rho}^{e}(\eta)\right\rangle_{\phi} \\
&-\int d \phi P(\phi)\left\langle\hat{\rho}^{e}(\epsilon)\right\rangle_{\phi} \int d \xi P(\xi)\left\langle\hat{\rho}^{e}(\eta)\right\rangle_{\xi}
\end{aligned}
$$

As shown in Appendix A, the form of Eq. (33) reflects the property that the probability distribution function $P(\phi)$ of the value $\phi$ of the function $\Phi(\psi)$ is unchanged during the process of solute insertion. Equation (34) is similar in structure to the blocking term in the theory of fluid in confined medium. ${ }^{17-20}$ The blocking term expresses the correlation between different replicas within the framework of the replica method. In the developments of Appendix A, it is not necessary to treat Eq. (34) explicitly. Our approximate expression for $\Delta \tilde{\mu}$ is then listed as

$$
\widetilde{w}^{e}(\epsilon)=-k_{B} T \log \left(\frac{\rho^{e}(\epsilon)}{\widetilde{\rho}_{0}^{e}(\epsilon)}\right)-\epsilon,
$$




$$
\begin{aligned}
& \widetilde{w}_{0}^{e}(\epsilon)=-k_{B} T \int d \eta\left(\frac{\delta(\epsilon-\eta)}{\widetilde{\rho}_{0}^{e}(\epsilon)}-\left(\widetilde{\chi}_{0}^{e}\right)^{-1}(\epsilon, \eta)\right)\left(\rho^{e}(\eta)-\widetilde{\rho}_{0}^{e}(\eta)\right), \\
& \Delta \widetilde{\mu}=-k_{B} T \int d \epsilon\left[\left(\rho^{e}(\epsilon)-\widetilde{\rho}_{0}^{e}(\epsilon)\right)+\beta \widetilde{w}^{e}(\epsilon) \rho^{e}(\epsilon)-\left\{\widetilde{\alpha}(\epsilon) \widetilde{F}(\epsilon)+(1-\widetilde{\alpha}(\epsilon)) \widetilde{F}_{0}(\epsilon)\right\}\left(\rho^{e}(\epsilon)-\widetilde{\rho}_{0}^{e}(\epsilon)\right)\right], \\
& \widetilde{F}(\epsilon)=\left\{\begin{array}{l}
\beta \widetilde{w}^{e}(\epsilon)+1+\frac{\beta \widetilde{w}^{e}(\epsilon)}{\exp \left(-\beta \widetilde{w}^{e}(\epsilon)\right)-1} \quad\left(\text { when } \widetilde{w}^{e}(\epsilon) \leqslant 0\right) \\
\frac{1}{2} \beta \widetilde{w}^{e}(\epsilon) \quad\left(\text { when } \widetilde{w}^{e}(\epsilon) \geqslant 0\right),
\end{array}\right. \\
& \widetilde{F}_{0}(\epsilon)=\left\{\begin{array}{l}
-\log \left(1-\beta \widetilde{w}_{0}^{e}(\epsilon)\right)+1+\frac{\log \left(1-\beta \widetilde{w}_{0}^{e}(\epsilon)\right)}{\beta \widetilde{w}_{0}^{e}(\epsilon)} \quad\left(\text { when } \widetilde{w}_{0}^{e}(\epsilon) \leqslant 0\right) \\
\frac{1}{2} \beta \widetilde{w}_{0}^{e}(\epsilon) \quad\left(\text { when } \widetilde{w}_{0}^{e}(\epsilon) \geqslant 0\right),
\end{array}\right. \\
& \widetilde{\alpha}(\epsilon)=\left\{\begin{array}{l}
1 \quad\left(\text { when } \rho^{e}(\epsilon) \geqslant \widetilde{\rho}_{0}^{e}(\epsilon)\right) \\
1-\left(\frac{\rho^{e}(\epsilon)-\widetilde{\rho}_{0}^{e}(\epsilon)}{\rho^{e}(\epsilon)+\widetilde{\rho}_{0}^{e}(\epsilon)}\right)^{2} \quad\left(\text { when } \rho^{e}(\epsilon) \leqslant \widetilde{\rho}_{0}^{e}(\epsilon)\right) .
\end{array}\right.
\end{aligned}
$$

This set of approximation is exact to second order in the solvent density and in the solute-solvent interaction for any choice of the function $\Phi(\psi){ }^{21}$ Equations (35) - (40) are coincident with Eqs. (13) - (18), furthermore, when $\Phi(\psi)$ is a constant independent of the solute intramolecular coordinate $\psi$.

In the present work, we set $\Phi(\psi)$ simply to the intramolecular potential function $\Psi(\psi)$ of the solute through

$$
\Phi(\psi)=\Psi(\psi) .
$$

The intramolecular potential for the reference solute molecule introduced by Eq. (21) then reduces to

$$
-k_{B} T \log \left(\frac{P(\Psi(\psi))}{\Omega_{0}(\Psi(\psi))}\right)
$$

within an additive constant independent of the solute intramolecular coordinate $\psi$, where $P$ is the distribution of the $\Psi$ value given by Eq. (19) and $\Omega_{0}$ is the density of states for the potential $\Psi$ expressed as

$$
\Omega_{0}(\phi)=\int d \psi \delta(\phi-\Psi(\psi)) .
$$

In summary, Eqs. (29) and (35)-(41) constitute the modified form of approximation in the present work. ${ }^{22}$

When the solute molecule is at isolation and is subject to the original form $\Psi$ of intramolecular potential, the conditional probability distribution function of the intramolecular coordinate $\psi$ is independent of $\psi$ at each fixed value of $\Psi$. Equation (22) then shows that when the function $\Phi$ is set to Eq. (41), the conditional distribution under a fixed $\Phi$ is also constant for the reference solute molecule. The conditional probability distribution function is thus always more localized in solution than at isolation under a particular choice expressed as Eq. (41). In Sec. IV B, we see that the performance of the original form of approximation does not deteriorate when the solute-solvent interaction localizes the structural distribution of the solute. Therefore, it is justified that the conditional probability distribution of $\psi$ at each fixed
$\Phi$ is not changed in the modification of the approximate scheme. The probability distribution function of the $\Phi$ value becomes common, on the other hand, to the solution system of interest and the reference solute molecule by adopting Eq. (41). The sampling scheme is then optimized straightforwardly with respect to the solvent effect on the solute intramolecular energy.

\section{PROCEDURES}

\section{A. System}

The purpose of Secs. III and IV is to illustrate the performance of the approach to the solvation free energy $\Delta \mu$ described in Sec. II. The accuracy of an approximate functional for $\Delta \mu$ under a given set of potential functions can be assessed through the exact evaluation of $\Delta \mu$ under the same set of potential functions. ${ }^{1,7}$ When such common techniques as the free energy perturbation and thermodynamic integration methods are used, ${ }^{1,7}$ however, the calculation of the exact $\Delta \mu$ is difficult in practice for a solute molecule with structural flexibility. For the purpose of obtaining the exact $\Delta \mu$, the number of intramolecular degrees of freedom needs to be small. In the present work, we employ a set of model solute molecules which involve a one-dimensional intramolecular coordinate. This is done so due to the limit of practical computational effort for calculating the exact $\Delta \mu$, although the method in Sec. II is developed to treat a solute molecule with intramolecular coordinates of any dimension.

The solvent is water. The water molecule is treated as rigid and nonpolarizable, and the SPC/E model is adopted as the intermolecular potential function between water molecules. ${ }^{23}$ Four thermodynamic states are then examined. One is an ambient state of $1.0 \mathrm{~g} / \mathrm{cm}^{3}$ and $25^{\circ} \mathrm{C}$ and the others are supercritical states of $1.0,0.6$, and $0.2 \mathrm{~g} / \mathrm{cm}^{3}$ and $400{ }^{\circ} \mathrm{C}$. In the following, the thermodynamic state of each system of interest is specified by the water density and temperature. 
The solute molecules employed in the present work are comprised of two interaction sites. In this case, the intramolecular potential of the solute molecule is determined by the distance $r$ between the sites. Two types of potential functions are adopted for the intramolecular degree of freedom. One is harmonic in the form given by ${ }^{24}$

$$
K\left(r-r_{e}\right)^{2},
$$

and the other is flat and is simply

$$
\begin{cases}0 & \left(\text { when }\left|r-r_{e}\right| \leqslant \Delta / 2\right) \\ \infty & \left(\text { when }\left|r-r_{e}\right|>\Delta / 2\right) .\end{cases}
$$

In both potential functions, $r_{e}$ is taken to be $3 \AA$. Furthermore, two values of 2 and $5 \mathrm{kcal} / \mathrm{mol} / \AA^{2}$ are examined for $K$ of Eq. (44), and 3 and $5 \AA$ for $\Delta$ of Eq. (45).

The intermolecular interaction between the solute and water molecules consists of the Lennard-Jones and Coulombic terms, as usual, and is given by Eq. (23) of Ref. 5 with the truncation factor $S(\mathbf{x})$ set to unity. The Lennard-Jones parameters for the two-site solute molecule are taken from Ref. 25. The values for the sodium ion in Table I of Ref. 25 are assigned to one of the sites, and those for the chloride ion to the other. The Lennard-Jones part of the solute-water potential function is then constructed by the standard LorentzBerthelot combining rule. ${ }^{1}$ For the Coulombic interaction, two cases are examined. One is the nonpolar case, in which no charge is given to any of the sites. In the other case, the solute is polar and the charge of +1 in the unit of elementary charge is placed at the $\mathrm{Na}^{+}$-like site. The $\mathrm{Cl}^{-}$-like site involves the corresponding negative charge, so that the molecule is neutral in total.

The number of solute molecules treated in the present work is 8 according to the form of intramolecular potential, the parameter in the potential, and the charges on the sites. Thus, a convention to specify the solute type is necessary for convenience in the rest of the paper. When Eq. (44) is adopted for the intramolecular potential function, the solute molecule is called with a prefix "HM." The nonpolar solute is then termed HM-2-0 and HM-5-0, respectively, when the parameter $K$ is set to 2 and $5 \mathrm{kcal} / \mathrm{mol} / \AA^{2}$. Similarly, the polar solute involves a +1 charge at the $\mathrm{Na}^{+}$-like site, and is denoted by HM-2-1 and HM-5-1, respectively, for $K=2$ and $5 \mathrm{kcal} / \mathrm{mol} / \AA^{2}$. When Eq. (45) is employed for the intramolecular potential, the solute molecule is labeled with a prefix "FL." In this case, the nonpolar solute is identified as FL-3-0 and FL-5-0, respectively, when $\Delta$ is taken to be 3 and $5 \AA$. The polar solute is FL-3-1 and FL-5-1 in compliance with the $\Delta$ value.

As described in Sec. III B, the electrostatic potential is handled by the Ewald method in the present work. A molecule then interacts with its own images when the molecule involves (partial) charges on its sites. This interaction is taken to be part of the intramolecular potential of the molecule concerned. ${ }^{26}$ In particular, the intramolecular energy of the HM-2-1 and HM-5-1 solutes is the sum of Eq. (44) and the interaction with the images, and is not exactly harmonic. For the FL-3-1 and FL-5-1 solutes, we actually set the (total) intramolecular potential to the form of Eq. (45). The interac- tion with the images is canceled so that the intramolecular potential is completely flat as a function of the site-site distance within the available range.

When a molecule involves two sites, its invariant measure for the relative coordinate of the intramolecular motion is $r^{2} d r d \Omega$, where $r$ is the radial distance between the sites and $d \Omega$ denotes the directional part. In the present work, the measure is instead taken to be $d r d \Omega$. This is done in order that the probability distribution function of $r$ for the solute at isolation (absence of the solute-solvent interaction) is simply proportional to the Boltzmann factor of the intramolecular energy without the $r^{2}$ factor of no interest for the purpose of assessing the performance of approximation. Especially, the $r$ distribution for the FL-3-0, FL-5-0, FL-3-1, and FL-5-1 solutes is constant within the accessible region of $r$ when no solvent is present.

\section{B. Simulation}

The inputs needed to evaluate the solvation free energy $\Delta \mu$ of the solute through Eqs. (29) and (35)-(41) are the distribution functions $\rho^{e}(\epsilon), \widetilde{\rho}_{0}^{e}(\epsilon)$, and $\widetilde{\chi}_{0}^{e}(\epsilon, \eta)$ introduced by Eqs. (8), (32), and (33), respectively. We obtain $\rho^{e}(\epsilon)$ from a Monte Carlo simulation of the solution system of interest, and $\widetilde{\rho}_{0}^{e}(\epsilon)$ and $\widetilde{\chi}_{0}^{e}(\epsilon, \eta)$ from a simulation of the pure solvent system. For comparison, we also calculate $\rho_{0}^{e}(\epsilon)$ and $\chi_{0}^{e}(\epsilon, \eta)$ given by Eqs. (10) and (11), respectively, and evaluate $\Delta \mu$ through the original form of approximation listed by Eqs. (13)-(18). It should be noted for the FL-3-0, FL-5-0, FL-3-1, and FL-5-1 solutes that $\widetilde{\rho}_{0}^{e}(\epsilon)$ and $\widetilde{\chi}_{0}^{e}(\epsilon, \eta)$ are the same as $\rho_{0}^{e}(\epsilon)$ and $\chi_{0}^{e}(\epsilon, \eta)$, respectively. For these solutes, the original and reference solute molecules introduced in Sec. II are identical to each other under a particular choice expressed as Eq. (41).

In each Monte Carlo simulation of the solution system, one solute molecule of interest and 300 water molecules were located in a cubic unit cell and the standard Metropolis sampling scheme in the canonical ensemble was implemented without the method of preferential sampling. ${ }^{7}$ The Monte Carlo simulation was performed for $50 \mathrm{~K}$ passes, where one pass corresponds to the generation of 300 configurations. The periodic boundary condition was employed in the minimum image convention, and the electrostatic potential was handled by the Ewald method with the surrounding medium of infinite dielectric constant. The screening parameter was then set to $5 / L$, where $L$ is the length of the unit cell, and 514 reciprocal lattice vectors were used. The truncation at $L / 2$ was applied on the site-site basis to the realspace part of the electrostatic interaction in the Ewald method and the Lennard-Jones part of the intermolecular interaction. The instantaneous distribution $\hat{\rho}^{e}(\epsilon)$ defined by Eq. (7) was sampled every pass. It was averaged through Eq. (8) over $50 \mathrm{~K}$ configurations of the solution system to construct the energy distribution function $\rho^{e}(\epsilon)$.

$\widetilde{\rho}_{0}^{e}(\epsilon)$ and $\widetilde{\chi}_{0}^{e}(\epsilon, \eta)$ were obtained by carrying out a Monte Carlo simulation of the pure solvent system. In the simulation, the standard Metropolis sampling scheme was implemented in the canonical ensemble by locating 300 water molecules in a cubic unit cell. The size of the unit cell 
was identical to that of the corresponding simulation of the solution system consisting of one solute molecule and 300 water molecules. The simulation length was $10 \mathrm{~K}$ passes, and the boundary condition and Ewald sum parameters were the same as those for the solution system. In parallel, a Monte Carlo simulation was performed for the solute molecule. The solute molecule was placed as a test particle in the unit cell of the neat solvent system, and did not affect the configuration of the solvent molecules. The position, orientation, and intramolecular state of the solute molecule were sampled through the standard Metropolis scheme. ${ }^{26}$ When the solute is HM-2-0, HM-5-0, HM-2-1, or HM-5-1, the intramolecular potential was modified from Eq. (42) in the actual Monte Carlo calculation for the reference solute molecule. It reads

$$
\left\{\begin{array}{l}
-k_{B} T \log \left(\frac{(1-q) P(\Psi(\psi))+q /\left(\phi_{\max }-\phi_{\min }\right)}{\Omega_{0}(\Psi(\psi))}\right) \quad\left(\text { when } \phi_{\min } \leqslant \Psi(\psi) \leqslant \phi_{\max }\right) \\
\infty \quad\left(\text { when } \Psi(\psi)<\phi_{\min } \text { or } \Psi(\psi)>\phi_{\max }\right),
\end{array}\right.
$$

where $q=0.1$ and $\phi_{\min }$ is the minimum possible value of the intramolecular energy $\Psi(\psi)$ of the solute molecule. $\phi_{\max }$ was set to $\left(\phi_{\min }+15 k_{B} T\right)$ for the HM-2-0 and HM-5-0 solutes and to $\left(\phi_{\min }+40 k_{B} T\right)$ for the HM-2-1 and HM-5-1, where $k_{B} T$ is the thermal energy. $P(\Psi(\psi))$ in Eq. (46) is introduced by Eq. (19) and can be obtained from the simulation of the corresponding solution system. $\Omega_{0}(\Psi(\psi))$ is simply the density of states given by Eq. (43) and its calculation is trivial for our model potentials. The modification was made in the present work because the equienergy surfaces are not connected for the solute intramolecular potential employed. When the solute is FL-3-0, FL-5-0, FL-3-1, or FL-5-1, its Monte Carlo simulation was conducted under the original form of intramolecular energy introduced by Eq. (45). In this case, the statistical weight for each intramolecular state of the solute at isolation (absence of the solute-solvent interaction) cannot be modified on the basis of the intramolecular energy, and Eq. (46) is different from Eq. (45) only within an additive constant. The instantaneous distribution $\hat{\rho}^{e}(\epsilon)$ was constructed by sampling an (instantaneous) configuration of the neat solvent system and an (instantaneous) state of the solute molecule present as a test particle. $\hat{\rho}^{e}(\epsilon)$ is the histogram for the interaction potential energies between the test solute particle and the solvent molecules, and was averaged to give $\widetilde{\rho}_{0}^{e}(\epsilon)$ and $\widetilde{\chi}_{0}^{e}(\epsilon, \eta)$ according to Eqs. (32) and (33). Of course, the method of umbrella sampling was utilized for the HM-2-0, HM-5-0, HM-2-1, and HM-5-1 solutes since the intramolecular energy for the Monte Carlo scheme was modified into Eq. (46). The configuration of the neat solvent system was sampled every 100 passes. At each solvent configuration used to calculate $\hat{\rho}^{e}(\epsilon)$, the solute molecule was sampled $5 \mathrm{~K}$ times with an interval of 50 steps of its Monte Carlo simulation. $\widetilde{\rho}_{0}^{e}(\epsilon)$ and $\widetilde{\chi}_{0}^{e}(\epsilon, \eta)$ were thus obtained from the averaging of $\hat{\rho}^{e}(\epsilon)$ over $500 \mathrm{~K}$ sets of solute and solvent configurations in a single simulation of the pure solvent system.

When the solute is HM-2-0, HM-5-0, HM-2-1, or HM$5-1, \quad \rho_{0}^{e}(\epsilon)$ and $\chi_{0}^{e}(\epsilon, \eta)$ are distinct from $\widetilde{\rho}_{0}^{e}(\epsilon)$ and $\widetilde{\chi}_{0}^{e}(\epsilon, \eta)$, respectively. In the calculation of $\rho_{0}^{e}(\epsilon)$ and $\chi_{0}^{e}(\epsilon, \eta)$, the intramolecular energy of the solute adopted for its Monte Carlo sampling is

$$
\left\{\begin{array}{l}
-k_{B} T \log \left(\frac{(1-q) P_{0}(\Psi(\psi))+q /\left(\phi_{\max }-\phi_{\min }\right)}{\Omega_{0}(\Psi(\psi))}\right) \quad\left(\text { when } \phi_{\min } \leqslant \Psi(\psi) \leqslant \phi_{\max }\right) \\
\infty \quad\left(\text { when } \Psi(\psi)<\phi_{\min } \text { or } \Psi(\psi)>\phi_{\max }\right),
\end{array}\right.
$$

where $P_{0}$ is the distribution function for the solute at isolation and is introduced by Eq. (20). Of course, the calculation of $P_{0}$ is trivial for the model potentials employed in the present work. The other parameters in Eq. (47) were set equal to their counterparts in Eq. (46). Except for the potential function of the solute, the procedure for obtaining $\rho_{0}^{e}(\epsilon)$ and $\chi_{0}^{e}(\epsilon, \eta)$ was the same as that for $\widetilde{\rho}_{0}^{e}(\epsilon)$ and $\widetilde{\chi}_{0}^{e}(\epsilon, \eta)$ described above.

One set of simulations to approximately evaluate the solvation free energy consists of two simulations. One is of the solution system, and the other is of the pure solvent system involving the solute molecule as a test particle. When the solute molecule is subject to the potential given by Eq. (46), the simulation of the solution system needs to be done first. $P(\Psi(\psi))$ is then provided as an output, and is used as an input for the subsequent simulation of the pure solvent system. For each solute and at each thermodynamic state, we performed six sets of simulations and estimated the average and error of the solvation free energy through the standard expressions shown by Eqs. (25) and (26) of Ref. 5.

In a simulation of the pure solvent system, the correlation matrices $\chi_{0}^{e}$ and $\widetilde{\chi}_{0}^{e}$ are obtained according to Eqs. (11) and (33). Their inversion is then needed through Eqs. (14) and (36), respectively, when the solvation free energy is to be 
evaluated. The inversion is not possible, however, when the number of solvent molecules interacting with the solute is invariant against the change in the configuration of the system. In this case, Eqs. (14) and (36) cannot be processed as they are, and their treatment is described in Appendix B. In the present work, all the solvent molecules, including those with zero solute-solvent interaction energy, are always counted in constructing the energy distribution functions. $\chi_{0}^{e}$ and $\tilde{\chi}_{0}^{e}$ calculated are then not invertible, and the procedure in Appendix B is employed to evaluate the solvation free energy. In addition, Appendix $\mathrm{C}$ provides the numerical schemes which supplement those in the Appendix of Ref. 5.

In order to assess the accuracy of an approximate procedure for the solvation free energy $\Delta \mu$ under a given set of potential functions, its exact evaluation is required under the same set of potential functions. ${ }^{1,7}$ As noted at the beginning of Sec. III A, however, the calculation of the exact $\Delta \mu$ is difficult for a solute molecule with structural flexibility. We circumvent this difficulty by resorting to Eq. (6). In Eq. (6), $p(\psi)$ is the probability distribution function of the solute intramolecular state $\psi$ in the solution of interest and is an output of the simulation of the solution system described above. $p_{0}(\psi)$ is the distribution for the solute molecule in the absence of the solute-solvent interaction and can be straightforwardly obtained for the model potentials in the present work. The (overall) solvation free energy $\Delta \mu$ can then be evaluated by calculating the solvation free energy $\Delta \sigma\left(\psi_{f}\right)$ at a fixed intramolecular state $\psi_{f}$ of the solute. In this scheme, the exact free energy calculation is necessary only for a rigid solute molecule with the structure identified by $\psi_{f} \cdot p\left(\psi_{f}\right)$ and $p_{0}\left(\psi_{f}\right)$ correct the difference between $\Delta \mu$ and $\Delta \sigma\left(\psi_{f}\right)$, so that $\psi_{f}$ is desirable to be "typical" in the solution system for achieving good precision of $\Delta \mu{ }^{27}$ In our treatments, $\psi_{f}$ was set to the site-site distance of $3.0 \AA$ for the HM-2-0, HM-5-0, FL-3-0, and FL-5-0 solutes. For the polar solutes, it was taken to be $4.5 \AA$ for the HM-2-1 and FL-3-1 solutes and $5.5 \AA$ for the HM-5-1 and FL-5-1.

The calculation of $\Delta \sigma\left(\psi_{f}\right)$ was performed by the free energy perturbation method. In each free energy calculation, a Monte Carlo simulation was carried out using the standard Metropolis sampling scheme in the canonical ensemble. One solute molecule of interest and 300 water molecules were then located in a cubic unit cell, and the preferential sampling method was not used. The size of the unit cell, the boundary condition, and the Ewald sum parameters were taken to be identical to those of the corresponding simulations of the solution and pure solvent systems.

In the free energy perturbation method, the solutesolvent interaction is controlled by the coupling parameter $\lambda(0 \leqslant \lambda \leqslant 1)$. For the nonpolar solutes, the Coulombic term is absent in the solute-solvent interaction. In this case, the Lennard-Jones term was turned on according to the expression obtained by replacing $\lambda$ of Eq. (27) of Ref. 5 with $\lambda / 3$. For the polar solutes, the Lennard-Jones and Coulombic terms were varied through Eqs. (27) and (28) of Ref. 5. In our calculations, the coupling parameter $\lambda$ was discretely changed in 50 and 90 steps for the nonpolar and polar solutes, respectively, with the equally spaced intervals. At each value of $\lambda$, the system was equilibrated for $5 \mathrm{~K}$ passes and the free energy change to the system at the next $\lambda$ was calculated for $5 \mathrm{~K}$ passes. The variation of $\lambda$ from 0 to 1 corresponds to the creation of the solute molecule and the reverse variation from 1 to 0 corresponds to the annihilation. We performed three sets of free energy perturbation calculations for both the creation and annihilation processes. Six values were then obtained for the solvation free energy, and the average and error were estimated by Eqs. (25) and (26) of Ref. 5.

\section{RESULTS AND DISCUSSION}

\section{A. Solute intramolecular structure}

In this section, we first describe the effect of solvent on the solute structure. The structure of the solute in solution is fully characterized by the probability distribution function $p$ of the intramolecular coordinate expressed as Eq. (5). The solvent effect is then seen from the comparison of $p$ with the distribution function $p_{0}$ for the solute at isolation (absence of the solute-solvent interaction) given by Eq. (4). In Fig. 1, we show $p$ and $p_{0}$ of the nonpolar solutes HM-2-0 and HM-5-0 as functions of the distance $r$ between the sites. It is evident for each solute and at each thermodynamic state that the solute-solvent interaction shifts the peak of the $r$ distribution to a shorter distance. In other words, the solute molecule becomes compact through its interaction with water, in agreement with the common notion of hydrophobicity. When the (solvent) density is fixed at $1.0 \mathrm{~g} / \mathrm{cm}^{3}$, the solvent effect on the $r$ distribution is apparently observed more strongly at a supercritical temperature of $400^{\circ} \mathrm{C}$ than at an ambient temperature of $25^{\circ} \mathrm{C}$. This observation reflects the fact that $K$ in Eq. (44) is taken to be invariant against the change in the thermodynamic state. The intramolecular potential of the sol-

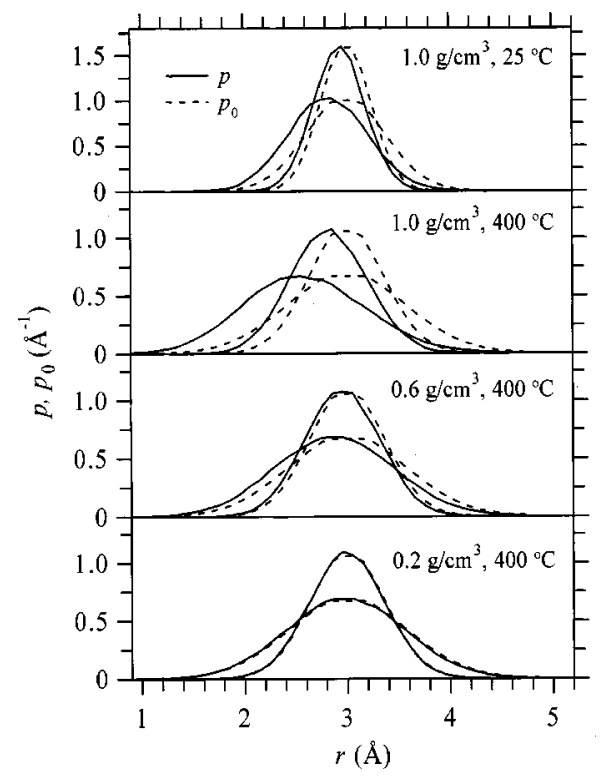

FIG. 1. The probability distribution functions $p$ and $p_{0}$ of the distance $r$ between the two sites of the HM-2-0 and HM-5-0 solutes at an ambient state of $1.0 \mathrm{~g} / \mathrm{cm}^{3}$ and $25^{\circ} \mathrm{C}$ and supercritical states of $1.0,0.6$, and $0.2 \mathrm{~g} / \mathrm{cm}^{3}$ and $400{ }^{\circ} \mathrm{C}$. For each of $p$ and $p_{0}$, the broader and sharper curves correspond to the HM-2-0 and HM-5-0 solutes, respectively. It should be noted that $p_{0}$ is common to the three supercritical states. 


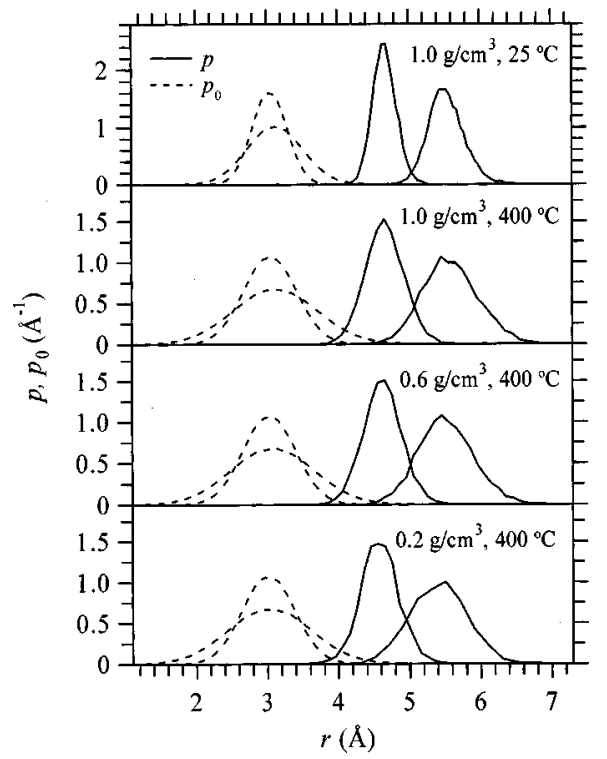

FIG. 2. The probability distribution functions $p$ and $p_{0}$ of the distance $r$ between the two sites of the HM-2-1 and HM-5-1 solutes at an ambient state of $1.0 \mathrm{~g} / \mathrm{cm}^{3}$ and $25^{\circ} \mathrm{C}$ and supercritical states of $1.0,0.6$, and $0.2 \mathrm{~g} / \mathrm{cm}^{3}$ and $400{ }^{\circ} \mathrm{C}$. For each of $p$ and $p_{0}$, the broader and sharper curves correspond to the HM-2-1 and HM-5-1 solutes, respectively. Due to the interaction of the solute with its own images, $p_{0}$ in the Ewald method depends slightly on the density even when the temperature is fixed.

ute is effectively "softer" at a higher temperature. When the temperature is fixed at $400{ }^{\circ} \mathrm{C}$, the density reduction leads to a weaker effect of solvation. Actually, $p$ and $p_{0}$ are barely distinguishable from each other at a low density of $0.2 \mathrm{~g} / \mathrm{cm}^{3}$.

The probability distribution functions $p$ and $p_{0}$ are shown in Fig. 2 for the polar solutes HM-2-1 and HM-5-1. It is observed for each case that the solute-solvent interaction favors the charge separation of the solute and gives rise to an extended structure. Actually, the solvent effect is strong and $p$ overlaps barely with $p_{0}$. The variation of the density and temperature leads only to a weak change in the peak position within the thermodynamic range examined, while the peak is broader at the higher temperature. Of course, $p$ reduces to $p_{0}$ in the limit of zero solvent density. The density of $0.2 \mathrm{~g} / \mathrm{cm}^{3}$ is then not "low enough" in the sense that $p$ at $0.2 \mathrm{~g} / \mathrm{cm}^{3}$ and $400{ }^{\circ} \mathrm{C}$ is closer to $p$ at the high-density states of $1.0 \mathrm{~g} / \mathrm{cm}^{3}$ and 25 and $400{ }^{\circ} \mathrm{C}$ than to $p_{0}$. This is consistent with a previous finding that the hydration of a polar or ionic species even at low-density supercritical states of $\sim 0.1 \mathrm{~g} / \mathrm{cm}^{3}$ is comparable to that at ambient states. ${ }^{28-30}$

For the FL-3-0, FL-5-0, FL-3-1, and FL-5-1 solutes, the solvent effect dominates the structural distribution function $p$ in solution since the distribution $p_{0}$ at isolation is constant within the accessible range of the site-site distance $r$ of the solute molecule. In Fig. 3, we show $p / p_{0}$ of these solutes. The behavior is parallel to that observed for the HM-2-0, HM-5-0, HM-2-1, and HM-5-1 solutes. When the solvent water is present, the nonpolar solute becomes compact and the polar solute is extended. For the FL-5-0 solute, $p / p_{0}$ reduces with the decrease of the distance $r$ in the small $r$ region. This reflects the property that the solute-solvent interaction is effectively less attractive when the two sites

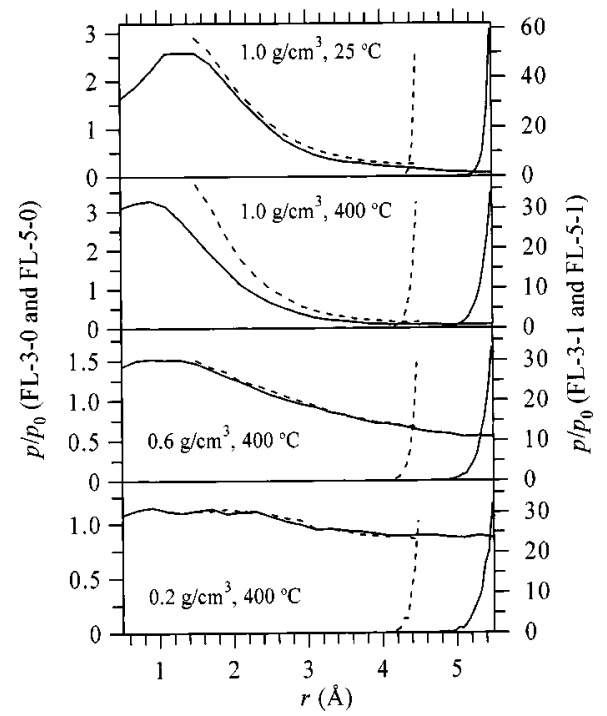

FIG. 3. The relative distribution function $p / p_{0}$ of the distance $r$ between the two sites of the FL-3-0, FL-5-0, FL-3-1, and FL-5-1 solutes at an ambient state of $1.0 \mathrm{~g} / \mathrm{cm}^{3}$ and $25^{\circ} \mathrm{C}$ and supercritical states of $1.0,0.6$, and $0.2 \mathrm{~g} / \mathrm{cm}^{3}$ and $400{ }^{\circ} \mathrm{C}$. The dashed curve with a sharp peak at $4.5 \AA$ represents the FL-3-1 solute and the other dashed curve corresponds to the FL3-0. The solid curve peaked at $5.5 \AA$ stands for the FL-5-1 solute and the other solid curve is for the FL-5-0. $p / p_{0}$ for the FL-3-0 and FL-5-0 solutes refer to the left ordinate, and those for the FL-3-1 and FL-5-1 to the right. For each solute and at each thermodynamic state, $p_{0}$ is constant in the accessible region of $r$ given by Eq. (45), and $p / p_{0}$ is not available outside the accessible region. Within a factor independent of $r, p / p_{0}$ is coincident between the FL-3-0 and FL-5-0 solutes and between the FL-3-1 and FL-5-1 by virtue of Eq. (6).

of the solute approach each other and their overlap is significant.

In Sec. II, a reduced form of description of the solute intramolecular state was introduced over the intramolecular potential energy $\phi$ in connection with the solvation free energy. The probability distribution function $P$ of $\phi$ in solution is derived from $p$ through a projection given by Eq. (19), and the distribution $P_{0}$ at isolation is from $p_{0}$ through Eq. (20). It is then insightful to illustrate the behaviors of $P$ and $P_{0}$ before discussion about the solvation free energy. In Fig. 4, $P$ and $P_{0}$ are shown for the HM-2-0 solute at the ambient state of $1.0 \mathrm{~g} / \mathrm{cm}^{3}$ and $25^{\circ} \mathrm{C}$ and the high-density supercritical state of $1.0 \mathrm{~g} / \mathrm{cm}^{3}$ and $400{ }^{\circ} \mathrm{C}$ and for the HM-2-1 solute at the ambient state and the low-density supercritical state of $0.2 \mathrm{~g} / \mathrm{cm}^{3}$ and $400{ }^{\circ} \mathrm{C}$. It should be noted that each of $P$ and $P_{0}$ diverges at the $\phi$ which is equal to an extremum of the intramolecular potential of the solute. This is caused by the corresponding divergence of the density of states expressed as Eq. (43) when the solute intramolecular degree of freedom is one-dimensional. The $\phi$ distribution is shifted to the higher-energy region by the solute-solvent interaction. Actually, the deviation of $P$ from $P_{0}$ is weak for the HM-2-0 solute, while the peak position is different by more than 10 $\mathrm{kcal} / \mathrm{mol}$ for the HM-2-1 solute.

The solvent effect on the distribution of the solute intramolecular energy is reflected in the solvation free energy through the second term of Eq. (29). The HM-2-1 and HM5-1 solutes are strongly affected by the solvent, and the second term of Eq. (29) amounts to $11-13 \mathrm{kcal} / \mathrm{mol}$. The term is 


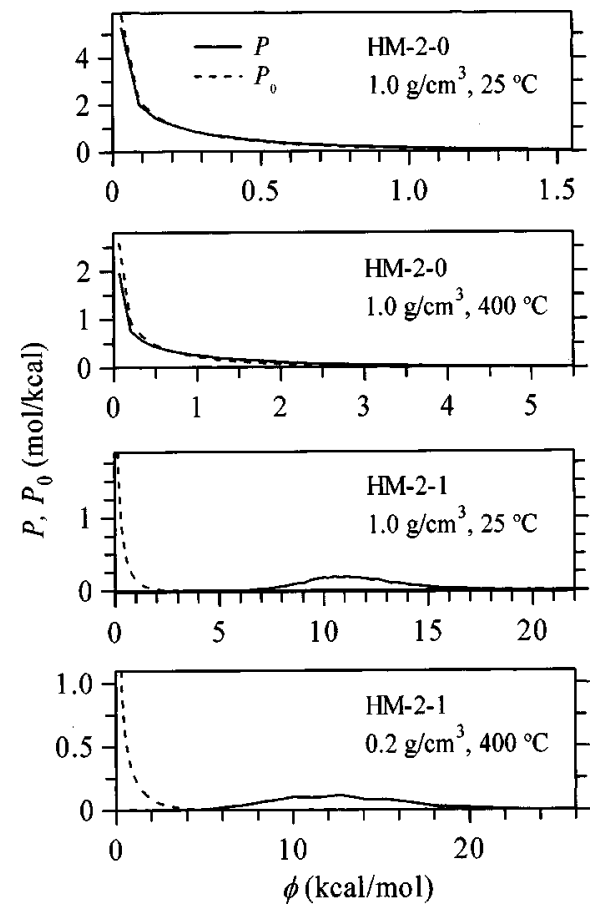

FIG. 4. The probability distribution functions $P$ and $P_{0}$ of the intramolecular potential energy $\phi$ of the HM-2-0 solute at an ambient state of $1.0 \mathrm{~g} / \mathrm{cm}^{3}$ and $25^{\circ} \mathrm{C}$ and a high-density supercritical state of $1.0 \mathrm{~g} / \mathrm{cm}^{3}$ and $400^{\circ} \mathrm{C}$ and of the HM-2-1 solute at the ambient state and a low-density supercritical state of $0.2 \mathrm{~g} / \mathrm{cm}^{3}$ and $400{ }^{\circ} \mathrm{C}$. The intramolecular potential is not coincident between the HM-2-0 and HM-2-1 solutes since the polar solute interacts with its own images in the Ewald method. $\phi$ is set to zero at the minimum of the intramolecular potential, and each of $P$ and $P_{0}$ diverges at the minimum $\phi$.

smaller than $0.1 \mathrm{kcal} / \mathrm{mol}$, on the other hand, when the solute is nonpolar and is HM-2-0 or HM-5-0. In this case, the distribution function of the solute intramolecular energy responds weakly to the solvation and the solvation free energy is dominated by the first term of Eq. (29).

\section{B. Solvation free energy}

In Sec. II, we presented two forms of approximation to the solvation free energy $\Delta \mu$. One is the original form formulated in Ref. 5 and is written as Eqs. (13)-(18). The other is a modified form and is given by Eqs. (29) and (35)-(41). The two approximate values for $\Delta \mu$ of the HM-2-0, HM-5-0, HM-2-1, and HM-5-1 solutes are listed in Table I and are compared to the exact values obtained from the free energy calculations. According to Table I, the $\Delta \mu$ values evaluated from the modified form of approximation are in good agreement with the corresponding exact values. Therefore, the single functional expressed as Eqs. (29) and (35)-(41) provides an efficient and accurate route to the solvation free energy of a flexible solute molecule over a wide range of thermodynamic conditions.

For the HM-2-0 and HM-5-0 solutes, however, the $\Delta \mu$ values obtained from the modified form of approximation are essentially coincident with the corresponding values calculated from the original form of approximation given by Eqs. (13)-(18). The solvation free energies of these solutes are not affected by the modification of the approximation. Numerical troubles were observed, on the other hand, when the original form of approximation is employed to calculate $\Delta \mu$ for the polar solutes HM-2-1 and HM-5-1. $\Delta \mu$ for the polar solute could not be determined due to numerical instability or was estimated only roughly with a discretization error typically of a few $\mathrm{kcal} / \mathrm{mol}$. Thus, the observation for the HM-2-1 and HM-5-1 solutes limits the utility of Eqs. (13) $-(18) .^{31}$

To see the nature of the modification of the approximate scheme, it is insightful to examine the FL-3-0, FL-5-0, FL3-1, and FL-5-1 solutes. Indeed, $\Delta \mu$ of these solutes are not affected by the modification of the approximation and are helpful to characterize the choice specified by Eq. (41). The approximate values of $\Delta \mu$ are shown in Table I and are found to agree with the corresponding exact values. The connection with the solvent effect on the solute structure is then seen from the distribution function of the solute intramolecular coordinate $\psi$. When the distribution function $p$ in the solution expressed as Eq. (5) is compared to the distribution function $p_{0}$ at isolation given by Eq. (4), it is illustrated in Fig. 3 that $p$ is more localized in the $\psi$ space than $p_{0}$. The localization is actually inevitable when the intramolecular potential of the solute takes the form of Eq. (45) and is constant within the accessible region of $\psi$. Table I thus demonstrates that when the presence of solvent localizes the structural distribution of the solute, the performance of the approximate scheme does not deteriorate in its original form listed by Eqs. (13)-(18). Our choice of Eq. (41) relies on the fact that the conditional probability distribution function of $\psi$ for the solute at isolation does not depend on $\psi$ at each fixed value of the intramolecular potential. When Eq. (41) is adopted, the conditional distribution is always more localized in solution than at isolation. The approximate scheme is then necessary to be optimized with respect only to the solvent effect on the solute intramolecular energy, and Eq. (41) is the choice to meet this necessity.

For the HM-2-0, HM-5-0, HM-2-1, and HM-5-1 solutes, the effect of the modification of the approximate scheme is clarified by comparing the distribution function of the solute intramolecular energy in the solution with that at isolation. When the solute is nonpolar, the distribution function responds weakly to the introduction of the solute-solvent interaction, as illustrated in Fig. 4. In this case, the modification presented in Sec. II causes only a minor revision of the sampling scheme for the solute intramolecular coordinate and does not lead to improvement of the approximation. When the solute is polar, in contrast, the intramolecular energy of the solute changes significantly through the interaction with the solvent and the performance of the approximate scheme is improved by employing the modified form.

\section{Solute-solvent interaction}

The modified form of approximation for the solvation free energy was formulated in Sec. II by introducing the notion of reference solute molecule. The reference solute molecule adopts some structural characteristics of the solute in the solution of interest, while it is to be placed, like the 
TABLE I. Solvation free energy in units of $\mathrm{kcal} / \mathrm{mol} .^{\mathrm{a}}$

\begin{tabular}{|c|c|c|c|c|}
\hline \multirow[t]{2}{*}{ Solute } & \multirow[t]{2}{*}{ Thermodynamic state } & \multirow[t]{2}{*}{ Exact } & \multicolumn{2}{|c|}{ Approximate } \\
\hline & & & $\begin{array}{c}\text { Original form } \\
\text { [Eqs. (13)-(18)] }\end{array}$ & $\begin{array}{l}\text { Modified form } \\
\text { [Eqs. (29) and (35)-(41)] }\end{array}$ \\
\hline \multirow[t]{4}{*}{ HM-2-0 } & $1.0 \mathrm{~g} / \mathrm{cm}^{3}$ and $25^{\circ} \mathrm{C}$ & $3.2 \pm 0.2$ & $3.5 \pm 0.1$ & $3.4 \pm 0.1$ \\
\hline & $1.0 \mathrm{~g} / \mathrm{cm}^{3}$ and $400^{\circ} \mathrm{C}$ & $15.1 \pm 0.3$ & $12.4 \pm 0.1$ & 12.5 \\
\hline & $0.6 \mathrm{~g} / \mathrm{cm}^{3}$ and $400^{\circ} \mathrm{C}$ & $3.5 \pm 0.1$ & $3.9 \pm 0.1$ & 3.9 \\
\hline & $0.2 \mathrm{~g} / \mathrm{cm}^{3}$ and $400^{\circ} \mathrm{C}$ & $0.7 \pm 0.1$ & 0.7 & 0.7 \\
\hline \multirow[t]{4}{*}{ HM-5-0 } & $1.0 \mathrm{~g} / \mathrm{cm}^{3}$ and $25^{\circ} \mathrm{C}$ & $3.3 \pm 0.2$ & $3.6 \pm 0.1$ & $3.4 \pm 0.2$ \\
\hline & $1.0 \mathrm{~g} / \mathrm{cm}^{3}$ and $400^{\circ} \mathrm{C}$ & $15.3 \pm 0.3$ & $12.6 \pm 0.1$ & $12.6 \pm 0.1$ \\
\hline & $0.6 \mathrm{~g} / \mathrm{cm}^{3}$ and $400^{\circ} \mathrm{C}$ & $3.5 \pm 0.1$ & 3.9 & 3.9 \\
\hline & $0.2 \mathrm{~g} / \mathrm{cm}^{3}$ and $400^{\circ} \mathrm{C}$ & $0.7 \pm 0.1$ & 0.7 & 0.7 \\
\hline \multirow[t]{4}{*}{ HM-2-1 } & $1.0 \mathrm{~g} / \mathrm{cm}^{3}$ and $25^{\circ} \mathrm{C}$ & $-104.8 \pm 0.9$ & $\ldots \mathrm{b}$ & $-108.5 \pm 1.8$ \\
\hline & $1.0 \mathrm{~g} / \mathrm{cm}^{3}$ and $400^{\circ} \mathrm{C}$ & $-86.2 \pm 0.5$ & $\ldots \mathrm{b}$ & $-87.2 \pm 0.5$ \\
\hline & $0.6 \mathrm{~g} / \mathrm{cm}^{3}$ and $400^{\circ} \mathrm{C}$ & $-86.4 \pm 0.3$ & $\ldots \mathrm{b}$ & $-82.9 \pm 0.4$ \\
\hline & $0.2 \mathrm{~g} / \mathrm{cm}^{3}$ and $400^{\circ} \mathrm{C}$ & $-78.6 \pm 1.3$ & $\ldots \mathrm{b}$ & $-70.9 \pm 0.4$ \\
\hline \multirow[t]{4}{*}{ HM-5-1 } & $1.0 \mathrm{~g} / \mathrm{cm}^{3}$ and $25^{\circ} \mathrm{C}$ & $-92.5 \pm 0.7$ & $\ldots \mathrm{b}$ & $-97.5 \pm 1.7$ \\
\hline & $1.0 \mathrm{~g} / \mathrm{cm}^{3}$ and $400^{\circ} \mathrm{C}$ & $-74.1 \pm 0.7$ & $\ldots \mathrm{b}$ & $-74.3 \pm 0.2$ \\
\hline & $0.6 \mathrm{~g} / \mathrm{cm}^{3}$ and $400^{\circ} \mathrm{C}$ & $-74.7 \pm 0.5$ & $\ldots \mathrm{b}$ & $-70.5 \pm 0.3$ \\
\hline & $0.2 \mathrm{~g} / \mathrm{cm}^{3}$ and $400^{\circ} \mathrm{C}$ & $-67.4 \pm 1.0$ & $\ldots \mathrm{b}$ & $-60.8 \pm 0.7$ \\
\hline \multirow[t]{4}{*}{ FL-3-0 ${ }^{c}$} & $1.0 \mathrm{~g} / \mathrm{cm}^{3}$ and $25^{\circ} \mathrm{C}$ & $2.9 \pm 0.2$ & $3.3 \pm 0.1$ & \\
\hline & $1.0 \mathrm{~g} / \mathrm{cm}^{3}$ and $400^{\circ} \mathrm{C}$ & $14.6 \pm 0.3$ & 12.1 & \\
\hline & $0.6 \mathrm{~g} / \mathrm{cm}^{3}$ and $400^{\circ} \mathrm{C}$ & $3.4 \pm 0.2$ & 3.8 & \\
\hline & $0.2 \mathrm{~g} / \mathrm{cm}^{3}$ and $400{ }^{\circ} \mathrm{C}$ & $0.6 \pm 0.2$ & 0.6 & \\
\hline \multirow[t]{4}{*}{ FL-5-0 ${ }^{c}$} & $1.0 \mathrm{~g} / \mathrm{cm}^{3}$ and $25^{\circ} \mathrm{C}$ & $2.9 \pm 0.3$ & $3.1 \pm 0.1$ & \\
\hline & $1.0 \mathrm{~g} / \mathrm{cm}^{3}$ and $400^{\circ} \mathrm{C}$ & $14.1 \pm 0.5$ & $11.6 \pm 0.1$ & \\
\hline & $0.6 \mathrm{~g} / \mathrm{cm}^{3}$ and $400^{\circ} \mathrm{C}$ & $3.4 \pm 0.4$ & 3.7 & \\
\hline & $0.2 \mathrm{~g} / \mathrm{cm}^{3}$ and $400{ }^{\circ} \mathrm{C}$ & $0.6 \pm 0.2$ & 0.6 & \\
\hline \multirow[t]{4}{*}{ FL-3-1 ${ }^{\mathrm{c}}$} & $1.0 \mathrm{~g} / \mathrm{cm}^{3}$ and $25^{\circ} \mathrm{C}$ & $-100.2 \pm 0.7$ & $-104.0 \pm 0.8$ & \\
\hline & $1.0 \mathrm{~g} / \mathrm{cm}^{3}$ and $400^{\circ} \mathrm{C}$ & $-79.7 \pm 0.7$ & $-80.4 \pm 0.2$ & \\
\hline & $0.6 \mathrm{~g} / \mathrm{cm}^{3}$ and $400^{\circ} \mathrm{C}$ & $-80.8 \pm 0.6$ & $-77.4 \pm 0.3$ & \\
\hline & $0.2 \mathrm{~g} / \mathrm{cm}^{3}$ and $400{ }^{\circ} \mathrm{C}$ & $-73.4 \pm 1.3$ & $-68.0 \pm 0.5$ & \\
\hline \multirow[t]{4}{*}{ FL-5- $1^{\mathrm{c}}$} & $1.0 \mathrm{~g} / \mathrm{cm}^{3}$ and $25^{\circ} \mathrm{C}$ & $-113.3 \pm 0.9$ & $-119.7 \pm 1.8$ & \\
\hline & $1.0 \mathrm{~g} / \mathrm{cm}^{3}$ and $400^{\circ} \mathrm{C}$ & $-92.5 \pm 0.3$ & $-94.2 \pm 0.5$ & \\
\hline & $0.6 \mathrm{~g} / \mathrm{cm}^{3}$ and $400^{\circ} \mathrm{C}$ & $-93.4 \pm 0.3$ & $-90.1 \pm 0.4$ & \\
\hline & $0.2 \mathrm{~g} / \mathrm{cm}^{3}$ and $400^{\circ} \mathrm{C}$ & $-86.2 \pm 1.3$ & $-80.6 \pm 0.9$ & \\
\hline
\end{tabular}

${ }^{a}$ Each value is rounded to a multiple of $0.1 \mathrm{kcal} / \mathrm{mol}$. The error is smaller than $0.1 \mathrm{kcal} / \mathrm{mol}$ when it is not shown.

${ }^{b}$ The value calculated from the original form of approximation is numerically unstable or involves a large discretization error typically of a few $\mathrm{kcal} / \mathrm{mol}$.

${ }^{c}$ The approximate value of the solvation free energy is identical between the original and modified forms of approximation.

original solute molecule, in the neat solvent system as a test particle. It is then insightful to illustrate the (one-body) energy distribution function $\widetilde{\rho}_{0}^{e}(\epsilon)$ for the reference solute molecule given by Eq. (32) and to note the connection with $\rho^{e}(\epsilon)$ for the solute molecule in solution and $\rho_{0}^{e}(\epsilon)$ for the original solute molecule expressed as Eqs. (8) and (10), respectively. In this section, we describe the behaviors of $\rho^{e}(\epsilon), \rho_{0}^{e}(\epsilon)$, and $\tilde{\rho}_{0}^{e}(\epsilon)$ for typical cases.

In Fig. 5, we show $\rho^{e}(\epsilon), \rho_{0}^{e}(\epsilon)$, and $\widetilde{\rho}_{0}^{e}(\epsilon)$ of the HM2-0 solute as functions of the solute-solvent interaction energy $\epsilon$ at the ambient state of $1.0 \mathrm{~g} / \mathrm{cm}^{3}$ and $25^{\circ} \mathrm{C}$ and the high-density supercritical state of $1.0 \mathrm{~g} / \mathrm{cm}^{3}$ and $400{ }^{\circ} \mathrm{C}$. It is evident for the nonpolar solute that $\rho_{0}^{e}(\epsilon)$ and $\tilde{\rho}_{0}^{e}(\epsilon)$ are barely distinguishable from each other within the precision of the figure. This behavior is simply related to the observation in Sec. IV A for the structural distribution function. When the solute is nonpolar, the solvent effect on the intramolecular structure is relatively weak. From the indistinguishability of $\rho_{0}^{e}(\epsilon)$ and $\tilde{\rho}_{0}^{e}(\epsilon)$, it is also natural for the nonpolar solute that the solvation free energy evaluated from the modified form of approximation is essentially coincident with that calculated from the original form of approximation. Of course, $\rho^{e}(\epsilon)$ is more populated in the favorable region of the solute-solvent interaction than $\rho_{0}^{e}(\epsilon)$ and $\widetilde{\rho}_{0}^{e}(\epsilon)$, and vanishes when the solute-solvent interaction is strongly unfavorable. $^{32}$

In Fig. 6, $\rho^{e}(\epsilon), \rho_{0}^{e}(\epsilon)$, and $\tilde{\rho}_{0}^{e}(\epsilon)$ are shown for the HM-2-1 solute at the ambient state of $1.0 \mathrm{~g} / \mathrm{cm}^{3}$ and $25^{\circ} \mathrm{C}$ 


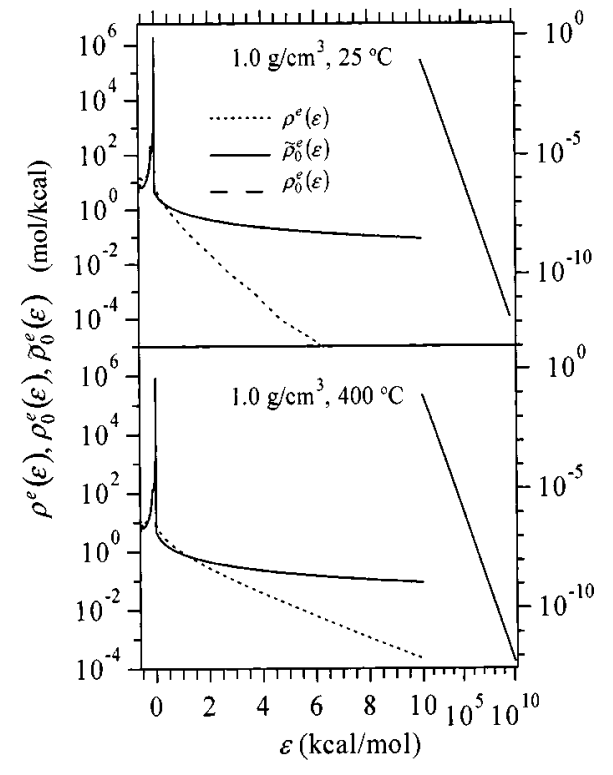

FIG. 5. The energy distribution functions $\rho^{e}(\epsilon), \rho_{0}^{e}(\epsilon)$, and $\widetilde{\rho}_{0}^{e}(\epsilon)$ of the HM-2-0 solute as functions of the energy coordinate $\epsilon$ at an ambient state of $1.0 \mathrm{~g} / \mathrm{cm}^{3}$ and $25^{\circ} \mathrm{C}$ and a high-density supercritical state of $1.0 \mathrm{~g} / \mathrm{cm}^{3}$ and $400^{\circ} \mathrm{C}$. When $\epsilon \leqslant 10 \mathrm{kcal} / \mathrm{mol}$, the abscissa is linearly graduated and the ordinate refers to the left. When $\epsilon \geqslant 10 \mathrm{kcal} / \mathrm{mol}$, only $\rho_{0}^{e}(\epsilon)$ and $\widetilde{\rho}_{0}^{e}(\epsilon)$ are shown with respect to the logarithmic abscissa and the right ordinate. The graduation for the ordinate is logarithmic for both the left and right. Actually, $\rho_{0}^{e}(\epsilon)$ and $\widetilde{\rho}_{0}^{e}(\epsilon)$ are barely distinguishable from each other within the precision of the figure.

and the low-density supercritical state of $0.2 \mathrm{~g} / \mathrm{cm}^{3}$ and $400{ }^{\circ} \mathrm{C}$. It is seen at the ambient state that $\rho^{e}(\epsilon)$ involves peaks at $\epsilon \simeq-33,-23$, and $-10 \mathrm{kcal} / \mathrm{mol}$. The last two peaks correspond to the "individual" binding of water with

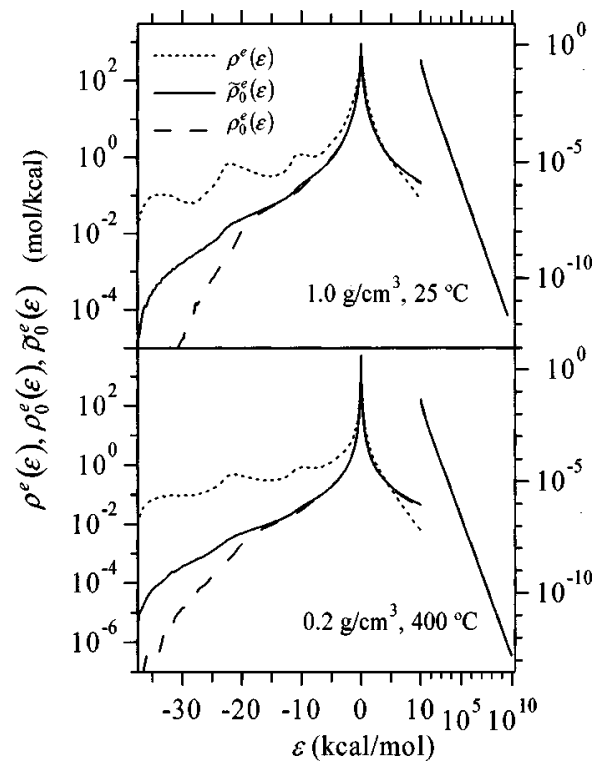

FIG. 6. The energy distribution functions $\rho^{e}(\epsilon), \rho_{0}^{e}(\epsilon)$, and $\widetilde{\rho}_{0}^{e}(\epsilon)$ of the HM-2-1 solute as functions of the energy coordinate $\epsilon$ at an ambient state of $1.0 \mathrm{~g} / \mathrm{cm}^{3}$ and $25^{\circ} \mathrm{C}$ and a low-density supercritical state of $0.2 \mathrm{~g} / \mathrm{cm}^{3}$ and $400^{\circ} \mathrm{C}$. When $\epsilon \leqslant 10 \mathrm{kcal} / \mathrm{mol}$, the abscissa is linearly graduated and the ordinate refers to the left. When $\epsilon \geqslant 10 \mathrm{kcal} / \mathrm{mol}$, only $\rho_{0}^{e}(\epsilon)$ and $\widetilde{\rho}_{0}^{e}(\epsilon)$ are shown with respect to the logarithmic abscissa and the right ordinate. The graduation for the ordinate is logarithmic for both the left and right. Within the precision of the figure, $\rho_{0}^{e}(\epsilon)$ and $\widetilde{\rho}_{0}^{e}(\epsilon)$ in the positive $\epsilon$ region are barely distinguishable from each other. the $\mathrm{Na}^{+}$-like and $\mathrm{Cl}^{-}$-like sites, respectively. The lowestenergy peak is present due to the water molecules interacting strongly with both the sites. When the temperature is elevated to the supercritical, the peak structure becomes less definite. The lowest-energy peak turns into a shoulder and the higher-energy peaks persist in broader forms.

Unlike the cases for the nonpolar solutes, $\rho_{0}^{e}(\epsilon)$ and $\tilde{\rho}_{0}^{e}(\epsilon)$ for the HM-2-1 and HM-5-1 solutes are distinct from each other in the favorable region of the solute-solvent interaction energy $\epsilon$. This reflects the observation in Sec. IV A that the intramolecular structure of the polar solute is strongly affected by the solvent. Actually, $\rho_{0}^{e}(\epsilon)$ in the lowest-energy side of $\epsilon \lessgtr-25 \mathrm{kcal} / \mathrm{mol}$ is only poorly sampled and deteriorates the performance of the original form of approximation. In the unfavorable region of the solute-solvent interaction $\epsilon$, the difference between $\rho_{0}^{e}(\epsilon)$ and $\widetilde{\rho}_{0}^{e}(\epsilon)$ is seen to be weak in Fig. 6. Thus, the indistinguishability of $\rho_{0}^{e}(\epsilon)$ and $\widetilde{\rho}_{0}^{e}(\epsilon)$ in the positive $\epsilon$ region is common to the nonpolar and polar solutes examined. ${ }^{32}$

\section{ACKNOWLEDGMENTS}

This work is supported by the Grant-in-Aid for Scientific Research (Nos. 11740322, 13640509, and 15205004) from the Japan Society for the Promotion of Science and by the Grant-in-Aid for Scientific Research on Priority Areas (No. 15076205) and the NAREGI (National Research Grid Initiative) Project from the Ministry of Education, Culture, Sports, Science, and Technology. N.M. is also grateful to the Supercomputer Laboratory of Institute for Chemical Research, Kyoto University, for generous allocation of computation time.

\section{APPENDIX A: APPROXIMATION TO $\Delta \tilde{\mu}$}

In this Appendix, we formulate an approximate expression for $\Delta \tilde{\mu}$ introduced by Eq. (30). The formulation is performed in two steps. In the first step, the one-to-one correspondence between the solute-solvent interaction potential and the solvent distribution around the solute is established with respect to the average of the free energy (change) of the form Eq. (30). The second step is then to obtain the approximate expression through the developments similar to those presented in Ref. 5.

As done in Refs. 4 and 5, the value of the solute-solvent interaction $v$ of interest is adopted as the coordinate $\epsilon$ in the energy representation. The attention is then restricted to a set of potentials $u$ which are constant over equienergy surfaces of $v$. A potential function $u$ contained in this set may be considered to be defined over the energy coordinate $\epsilon$ and can be expressed as $u^{e}(\epsilon)$. Of course, $u$ depends on the intramolecular coordinate $\psi$ of the solute and the full coordinate $\mathbf{x}$ of the solvent through the solute-solvent interaction $v$ of interest, and is denoted by $u^{f}(\psi, \mathbf{x})$ in the full coordinate representation. In Sec. II, a function $\Phi(\psi)$ defined over the coordinate $\psi$ is introduced to characterize the solute intramolecular state. When the value $\phi$ is fixed for $\Phi(\psi)$ in the presence of a solute-solvent interaction $u$, the average distribution $\rho^{e}(\epsilon, \phi ; u)$ is given in the energy representation by 


$$
\rho^{e}(\epsilon, \phi ; u)=\frac{\int d \psi d \mathbf{X} \delta(\phi-\Phi(\psi)) \hat{\rho}^{e}(\epsilon) \exp \left(-\beta\left\{\Psi(\psi)+\Sigma_{i} u^{f}\left(\psi, \mathbf{x}_{i}\right)+U(\mathbf{X})\right\}\right)}{\int d \psi d \mathbf{X} \delta(\phi-\Phi(\psi)) \exp \left(-\beta\left\{\Psi(\psi)+\Sigma_{i} u^{f}\left(\psi, \mathbf{x}_{i}\right)+U(\mathbf{X})\right\}\right)},
$$

where $\hat{\rho}^{e}$ is the instantaneous distribution defined as Eq. (7), $\mathbf{X}$ represents the solvent configuration collectively, and $\Psi(\psi)$ and $U(\mathbf{X})$ are the solute intramolecular energy and the solvent-solvent interaction energy, respectively. Correspondingly, when the solute molecule with the solute-solvent interaction $u$ is inserted at a fixed $\phi$, the free energy change $\Delta \tau(\phi ; u)$ is written as

$$
\exp (-\beta \Delta \tau(\phi ; u))=\frac{\int d \psi d \mathbf{X} \delta(\phi-\Phi(\psi)) \exp \left(-\beta\left\{\Psi(\psi)+\Sigma_{i} u^{f}\left(\psi, \mathbf{x}_{i}\right)+U(\mathbf{X})\right\}\right)}{\int d \psi d \mathbf{X} \delta(\phi-\Phi(\psi)) \exp (-\beta\{\Psi(\psi)+U(\mathbf{X})\})} .
$$

Obviously, $\Delta \tau(\phi ; u)$ reduces to $\Delta \nu(\phi)$ of Eq. (25) when its argument $u$ is set to the interaction potential $v$ of interest.

In our developments, the probability distribution function $P(\phi)$ of the value $\phi$ of the function $\Phi(\psi)$ is fixed at the one introduced by Eq. (19). The average distribution $\widetilde{\rho}^{e}(\epsilon ; u)$ and free energy change $\Delta \widetilde{\tau}(u)$ are then set, respectively, to

$$
\begin{aligned}
& \widetilde{\rho}^{e}(\epsilon ; u)=\int d \phi P(\phi) \rho^{e}(\epsilon, \phi ; u), \\
& \Delta \widetilde{\tau}(u)=\int d \phi P(\phi) \Delta \tau(\phi ; u) .
\end{aligned}
$$

$u$ is left as an argument in Eqs. (A3) and (A4) to specify the solute-solvent interaction potential. When $u$ is $v$ itself, $\widetilde{\rho}^{e}(\epsilon ; u)$ and $\Delta \widetilde{\tau}(u)$ are equal to $\rho^{e}(\epsilon)$ of Eq. (8) and $\Delta \tilde{\mu}$ of Eq. (30), respectively. When the solute-solvent interaction is absent $(u=0), \widetilde{\rho}^{e}(\epsilon ; u)$ is the distribution function in the pure solvent system with the reference solute molecule and is identical to $\widetilde{\rho}_{0}^{e}(\epsilon)$ given by Eq. (32). The response of $\widetilde{\rho}^{e}(\epsilon ; u)$ to the change in the solute-solvent interaction $u^{e}(\epsilon)$ is written as

$$
\begin{aligned}
\frac{\delta \widetilde{\rho}^{e}(\epsilon ; u)}{\delta\left(-\beta u^{e}(\eta)\right)}= & \int d \phi P(\phi)\left(\left\langle\hat{\rho}^{e}(\epsilon) \hat{\rho}^{e}(\eta)\right\rangle_{\phi ; u}\right. \\
& \left.-\rho^{e}(\epsilon, \phi ; u) \rho^{e}(\eta, \phi ; u)\right),
\end{aligned}
$$

where $\left\langle\hat{\rho}^{e}(\epsilon) \hat{\rho}^{e}(\eta)\right\rangle_{\phi ; u}$ is the expression obtained by replacing $\hat{\rho}^{e}(\epsilon)$ in the right-hand side of Eq. (A1) with $\hat{\rho}^{e}(\epsilon) \hat{\rho}^{e}(\eta)$. Equation (A5) shows that the second cumulant of $\hat{\rho}^{e}$ at fixed $\phi$ is averaged with the weight $P(\phi)$ to provide the response function. This type of average appears due to the property that $P(\phi)$ is unvaried within the systems concerned. Of course, Eq. (A5) reduces to Eq. (33) when $u=0$.

It is now straightforward to prove that the map is oneto-one from a set of potentials of the form $u^{e}(\epsilon)$ to the corresponding set of distribution functions $\widetilde{\rho}^{e}(\epsilon ; u)$. When two solute-solvent interactions $u$ and $w$ are provided, Eqs. (A1)-(A4) lead to

$$
\Delta \widetilde{\tau}(w)-\Delta \widetilde{\tau}(u) \leqslant \int d \epsilon\left(w^{e}(\epsilon)-u^{e}(\epsilon)\right) \widetilde{\rho}^{e}(\epsilon ; u),
$$

where the equality holds only when $\left(w^{e}(\epsilon)-u^{e}(\epsilon)\right)$ is a constant independent of $\epsilon$ and the number $N$ of solvent molecules interacting with the solute is invariant against the change in the configuration of the system. ${ }^{33}$ It then follows from an argument similar to the one described in Appendix B of Ref. 4 that different potentials $u^{e}(\epsilon)$ and $w^{e}(\epsilon)$ are mapped to different distributions $\widetilde{\rho}^{e}(\epsilon ; u)$ and $\widetilde{\rho}^{e}(\epsilon ; w)$ unless $u$ differs from $w$ by an additive constant in a system with invariable $N .{ }^{34}$ In Appendix B, we show a procedure to fix the additive constant. When this procedure is adopted, the map from the solute-solvent interaction to the distribution function is one-to-one in the energy representation. ${ }^{35}$

To obtain an approximate expression for $\Delta \tilde{\mu}$ given by Eq. (30), we treat the gradual insertion process of the solute. In the insertion process, the solute-solvent interaction is turned on according to the coupling parameter $\lambda(0 \leqslant \lambda$ $\leqslant 1)$. When $\lambda=0$, there is no explicit interaction between the solute and solvent and the system is the pure solvent with the reference solute molecule introduced in Sec. II. When $\lambda=1$, the solute interacts with the solvent at full coupling under the solute-solvent interaction potential $v$ of interest. In the energy representation, the gradual insertion of the solute is described by a family of solute-solvent interaction potentials $u_{\lambda}^{e}(\epsilon)$. Of course, it is imposed that

$$
\begin{aligned}
& u_{0}^{e}(\epsilon)=0, \\
& u_{1}^{e}(\epsilon)=v^{e}(\epsilon)=\epsilon .
\end{aligned}
$$

$\Delta \tilde{\mu}$ can then be expressed as

$$
\begin{aligned}
\Delta \tilde{\mu} & =\int_{0}^{1} d \lambda \int d \epsilon \frac{\partial u_{\lambda}^{e}(\epsilon)}{\partial \lambda} \widetilde{\rho}^{e}\left(\epsilon ; u_{\lambda}\right) \\
& =\int_{0}^{1} d \lambda \int d \phi \int d \epsilon \frac{\partial u_{\lambda}^{e}(\epsilon)}{\partial \lambda} P(\phi) \rho^{e}\left(\epsilon, \phi ; u_{\lambda}\right) .
\end{aligned}
$$

This equation is the charging formula for an average of the free energy (change) of the form Eq. (30) and is exact for any choice of $u_{\lambda}$. It should be noted that $P(\phi)$ appearing in Eq. (A8) does not change during the process of solute insertion.

As done in Ref. $5, u_{\lambda}$ is chosen so that $\widetilde{\rho}^{e}\left(\epsilon ; u_{\lambda}\right)$ varies linearly with $\lambda$. In other words,

$$
\widetilde{\rho}^{e}\left(\epsilon ; u_{\lambda}\right)=\lambda \rho^{e}(\epsilon)+(1-\lambda) \widetilde{\rho}_{0}^{e}(\epsilon)
$$

holds at each $\epsilon$ in our choice of $u_{\lambda}$, where $\rho^{e}(\epsilon)$ and $\widetilde{\rho}_{0}^{e}(\epsilon)$ are given by Eqs. (8) and (32), respectively. The unique existence of the $u_{\lambda}$ satisfying Eq. (A9) is assured by the oneto-one correspondence described above. To formulate the approximation, it is useful to employ the indirect part of the potential of mean force between the solute and solvent molecules. Within the context of this Appendix, the indirect part $\widetilde{w}^{e}$ of the solute-solvent potential of mean force is defined in the presence of a solute-solvent interaction $u$ as 


$$
\widetilde{w}^{e}(\epsilon ; u)=-k_{B} T \log \left(\frac{\widetilde{\rho}^{e}(\epsilon ; u)}{\widetilde{\rho}_{0}^{e}(\epsilon)}\right)-u^{e}(\epsilon) .
$$

When the argument $u$ is taken to be the interaction $v$ of interest, $\widetilde{w}^{e}(\epsilon ; v)$ is the same as $\widetilde{w}^{e}(\epsilon)$ introduced by Eq. (35). Under the particular choice of $u_{\lambda}$ identified by Eq. (A9), furthermore, $\widetilde{w}_{0}^{e}(\epsilon)$ of Eq. (36) is exactly related to $\widetilde{w}^{e}\left(\epsilon ; u_{\lambda}\right)$ through

$$
\widetilde{w}_{0}^{e}(\epsilon)=\left.\frac{\partial \widetilde{w}^{e}\left(\epsilon ; u_{\lambda}\right)}{\partial \lambda}\right|_{\lambda=0} .
$$

When the Percus-Yevick-type approximation is adopted along the $\lambda$ variation according to Eq. (A9), $\widetilde{w}^{e}\left(\epsilon ; u_{\lambda}\right)$ is expressed as

$$
\begin{aligned}
-\beta \widetilde{w}^{e}\left(\epsilon ; u_{\lambda}\right) & =\log \left(1+\lambda\left(\exp \left(-\beta \widetilde{w}^{e}(\epsilon)\right)-1\right)\right) \\
& =\log \left(1-\lambda \beta \widetilde{w}_{0}^{e}(\epsilon)\right) .
\end{aligned}
$$

The hypernetted-chain-type approximation is written, on the other hand, as

$$
\widetilde{w}^{e}\left(\epsilon ; u_{\lambda}\right)=\lambda \widetilde{w}^{e}(\epsilon)=\lambda \widetilde{w}_{0}^{e}(\epsilon) .
$$

The subsequent development is then a notational variant of that presented in Ref. 5, and leads to a set of approximate equations listed as Eqs. (35)-(40).

\section{APPENDIX B: INVERSION OF THE CORRELATION MATRIX}

The inverses of the correlation matrices $\chi_{0}^{e}(\epsilon, \eta)$ and $\widetilde{\chi}_{0}^{e}(\epsilon, \eta)$ are required, respectively, to determine $w_{0}^{e}(\epsilon)$ with Eq. (14) and $\widetilde{w}_{0}^{e}(\epsilon)$ with Eq. (36). The inversion is not possible, however, when the number of solvent molecules interacting with the solute is constant against the change in the configuration of the system. The purpose of this Appendix is to provide a scheme to determine $w_{0}^{e}(\epsilon)$ and $\widetilde{w}_{0}^{e}(\epsilon)$ when the inverses of the correlation matrices do not exist. The arguments are given only for $\chi_{0}^{e}(\epsilon, \eta)$ and $w_{0}^{e}(\epsilon)$ because those for $\widetilde{\chi}_{0}^{e}(\epsilon, \eta)$ and $\widetilde{w}_{0}^{e}(\epsilon)$ are parallel.

The number $N$ of solvent molecules interacting with the solute molecule at an instantaneous configuration of the system is expressed as

$$
N=\int d \epsilon \hat{\rho}^{e}(\epsilon)
$$

in terms of the instantaneous distribution $\hat{\rho}^{e}$ defined as Eq. (7). When the solute-solvent interaction is not truncated in a finite region, $N$ is simply the total number of solvent molecules in the system. $N$ is then independent of the system configuration when the ensemble employed does not allow the fluctuation of $N .{ }^{36}$ In this case,

$$
\int d \eta \chi_{0}^{e}(\epsilon, \eta)=0
$$

holds at each $\epsilon$. Equation (B2) shows that the correlation matrix involves a null eigenvalue and is not invertible. The eigenvector corresponding to the null eigenvalue can be identified by noting that

$$
\int d \epsilon d \eta g(\epsilon) \chi_{0}^{e}(\epsilon, \eta) g(\eta) \geqslant 0
$$

for any function $g(\epsilon)$ defined over the coordinate $\epsilon$. Since the left-hand side of Eq. (B3) is the variance of the sum of the $g$ values over all the solvent molecules, the equality is realized only when $N$ is invariable and $g(\epsilon)$ is a constant independent of $\epsilon$. Therefore, an eigenvector for $\chi_{0}^{e}(\epsilon, \eta)$ does not depend on the coordinate $\epsilon$ when the corresponding eigenvalue is zero. Especially, when two functions $g(\epsilon)$ and $h(\epsilon)$ satisfy

$$
\int d \eta \chi_{0}^{e}(\epsilon, \eta) g(\eta)=\int d \eta \chi_{0}^{e}(\epsilon, \eta) h(\eta),
$$

$g(\epsilon)$ differs from $h(\epsilon)$ by an additive constant.

When the truncation is applied to the solute-solvent interaction, the reduced form of instantaneous distribution is introduced by Eq. (8) of Ref. 4 and Eq. (22) of Ref. 5. In this case, the number of solvent molecules interacting with the solute varies in response to the change in the configuration of the system and the correlation matrix constructed from the reduced form of instantaneous distribution provides a nonzero value in the expression similar to Eq. (B3). All the eigenvalues are then positive for the correlation matrix, so that the inverse exists. As noted above, the inversion is not possible only when $N$ is fixed. In the rest of this Appendix, we restrict our attention to the case in which the correlation matrix is not invertible.

By employing an auxiliary function $\bar{u}_{0}^{e}$, Eq. (14) is rewritten as a set of equations through

$$
\begin{aligned}
& \int d \eta \chi_{0}^{e}(\epsilon, \eta) \bar{u}_{0}^{e}(\eta)=-k_{B} T\left(\rho^{e}(\epsilon)-\rho_{0}^{e}(\epsilon)\right), \\
& w_{0}^{e}(\epsilon)=-\bar{u}_{0}^{e}(\epsilon)-k_{B} T \frac{\rho^{e}(\epsilon)-\rho_{0}^{e}(\epsilon)}{\rho_{0}^{e}(\epsilon)} .
\end{aligned}
$$

Since $\chi_{0}^{e}(\epsilon, \eta)$ involves a null eigenvalue, the solution to Eq. (B5) expressed as $\bar{u}_{0}^{e}$ is not unique. From the above considerations, however, any two solutions are different only by an additive constant independent of the coordinate $\epsilon$. In other words, $\bar{u}_{0}^{e}$ can be uniquely determined when its value is fixed at a particular coordinate $\epsilon$.

In Ref. 5 and Appendix A of the present paper, the gradual insertion process of the solute is treated in terms of the coupling parameter $\lambda$ and is described by a family of solute-solvent interaction potentials $u_{\lambda}^{e}(\epsilon)$. Equation (13) of Ref. 5 then leads to

$$
\bar{u}_{0}^{e}(\epsilon)=\left.\frac{\partial u_{\lambda}^{e}(\epsilon)}{\partial \lambda}\right|_{\lambda=0}
$$

when the energy distribution function varies linearly with $\lambda$ through Eq. (11) of Ref. 5. Actually, the energy distribution function at each $\lambda$ is not affected by a constant shift of $u_{\lambda}^{e}(\epsilon)$. The origin of the potential has no effect on the distribution function when the number of solvent molecules interacting with the solute is invariable. This point is reflected in the fact that the solution is not unique in Eq. (B5) and is indeterminate up to an additive constant. 
We fix the additive constant on the basis of the physical argument concerning the intensive nature of the solvation free energy. The number of solvent molecules which are separated from the solute molecule by large distances increases in proportion to the total number of solvent molecules in the system. The solvation free energy then converges in the thermodynamic limit when the solute-solvent interaction potential vanishes at large distances. Of course, potential functions are always formulated to be zero at infinite separation. Thus, the solvation free energy is assured to be intensive for a common choice of interaction potential. Within the context of $u_{\lambda}^{e}(\epsilon)$, the solute-solvent interaction at large distances corresponds to the coordinate $\epsilon \approx 0$. Although $u_{\lambda}^{e}(\epsilon)$ at $\lambda \neq 0$ or 1 are not of physical interest, it is natural to require that the free energy change be intensive upon insertion of the solute molecule with the interaction $u_{\lambda}^{e}(\epsilon)$. This requirement then leads to

$$
u_{\lambda}^{e}=0 \text { at } \epsilon=0 .
$$

From Eqs. (B7) and (B8), we fix the additive constant by

$$
\bar{u}_{0}^{e}=0 \text { at } \epsilon=0 \text {. }
$$

When the solute-solvent interaction of interest is continuous, the energy coordinate $\epsilon$ needs to be discretized in the numerical implementation. The discretized version of Eq. (B5) reads

$$
\sum_{j} \delta_{j} \chi_{0}^{e}(i, j) \bar{u}_{0}^{e}(j)=-k_{B} T\left(\rho^{e}(i)-\rho_{0}^{e}(i)\right),
$$

where $\delta_{j}$ is the length of the $j$ th interval of the energy coordinate and $\chi_{0}^{e}(i, j), \bar{u}_{0}^{e}(i), \rho^{e}(i)$, and $\rho_{0}^{e}(j)$ are the discretized forms of $\chi_{0}^{e}(\epsilon, \eta), \bar{u}_{0}^{e}(\epsilon), \rho^{e}(\epsilon)$, and $\rho_{0}^{e}(\epsilon)$, respectively. Let $z_{i}$ be an eigenvalue for the symmetric matrix $\delta_{i} \chi_{0}^{e}(i, j) \delta_{j}$ and $g_{i}(j)$ be the corresponding eigenvector. The smallest eigenvalue, which we call $z_{0}$, is zero and is nondegenerate. When the set of eigenvectors is taken to be orthonormal, Eq. (B10) is solved as

$$
\bar{u}_{0}^{e}(i)=C-k_{B} T \sum_{j \neq 0} g_{j}(i) \frac{1}{z_{j}} \sum_{l} \delta_{l} g_{j}(l)\left(\rho^{e}(l)-\rho_{0}^{e}(l)\right),
$$

where the term corresponding to the null eigenvalue is omitted in the sum and $C$ is a constant independent of the discretized coordinate. Of course, $C$ is fixed, in accordance with Eq. (B9), by setting $\bar{u}_{0}^{e}(I)$ to be zero at the interval $I$ which contains $\epsilon=0$.

In the practical implementation, the eigenvalues $z_{i}$ and the eigenvectors $g_{i}(j)$ are always calculated with numerical errors. When the error of $z_{i}$ and $g_{i}(j)$ is of order $\kappa$, each term in the sum of Eq. (B11) involves an error of order $\kappa$. Due to the numerical error, in particular, the smallest eigenvalue $z_{0}$ may not be apparently zero. In this case, it is possible at the level of numerical manipulation to include the $j=0$ term in the sum of Eq. (B11). The $j=0$ term is of order 1 since the inner product [sum over $l$ in Eq. (B11)] between the eigenvector $g_{0}(l)$ and $\left(\rho^{e}(l)-\rho_{0}^{e}(l)\right)$ is zero in an exact calculation. Thus, the correct limit is not achieved at $\kappa \rightarrow 0$ if the $j=0$ term is included in the sum. Even when $\delta_{i} \chi_{0}^{e}(i, j) \delta_{j}$ is numerically invertible, the term corresponding to the smallest eigenvalue needs to be omitted in the sum of Eq. (B11).

\section{APPENDIX C: SUPPLEMENTARY NUMERICAL PROCEDURES}

The numerical procedures to treat the energy distribution functions are essentially described in the Appendix of Ref. 5 . In this Appendix, we present the numerical schemes which supplement those in Ref. 5. The presentation is made only for $\rho^{e}$ in the solution system and $\rho_{0}^{e}$ and $\chi_{0}^{e}$ in the pure solvent system. This is because the schemes are parallel for $\widetilde{\rho}_{0}^{e}$ and $\widetilde{\chi}_{0}^{e}$ calculated using the reference solute molecule.

In the present work, the number $N$ of solvent molecules interacting with the solute molecule is independent of the system configuration and does not fluctuate. It then follows by virtue of Eq. (B1) that the energy distribution functions used to evaluate the solvation free energy satisfy

$$
\begin{aligned}
& \int d \epsilon \rho^{e}(\epsilon)=N, \\
& \int d \epsilon \rho_{0}^{e}(\epsilon)=N, \\
& \int d \eta\left\langle\hat{\rho}^{e}(\epsilon) \hat{\rho}^{e}(\eta)\right\rangle_{0}=N \rho_{0}^{e}(\epsilon),
\end{aligned}
$$

where $\langle\cdots\rangle_{0}$ is the average in the pure solvent system given by Eq. (12) and $\left\langle\hat{\rho}^{e}(\epsilon) \hat{\rho}^{e}(\eta)\right\rangle_{0}$ provides $\chi_{0}^{e}(\epsilon, \eta)$ through Eq. (11). ${ }^{37}$ In an actual computer calculation, however, Eq. (C1) does not necessarily hold since the energy distribution functions are stored in finite digits. ${ }^{38}$ In our numerical implementation, the energy distribution functions constructed in the simulation are normalized when the solvation free energy is to be obtained. The normalization is simply to replace $\rho^{e}(\epsilon), \rho_{0}^{e}(\epsilon)$, and $\left\langle\hat{\rho}^{e}(\epsilon) \hat{\rho}^{e}(\eta)\right\rangle_{0}$ with $r_{1} \rho^{e}(\epsilon), r_{0} \rho_{0}^{e}(\epsilon)$, and $R(\epsilon) R(\eta)\left\langle\hat{\rho}^{e}(\epsilon) \hat{\rho}^{e}(\eta)\right\rangle_{0}$, respectively, where the factors $r_{1}, r_{0}$, and $R(\epsilon)$ are determined to enforce the condition Eq. (C1).

Even when the exact value of $\rho^{e}$ or $\rho_{0}^{e}$ is not zero at a particular energy coordinate $\epsilon_{I}$, it is sometimes calculated to be zero numerically in an actual simulation. ${ }^{39}$ It should be noted that when $\rho_{0}^{e}\left(\epsilon_{I}\right)$ is zero, $\chi_{0}^{e}\left(\epsilon_{I}, \eta\right)$ and $\chi_{0}^{e}\left(\eta, \epsilon_{I}\right)$ are also zero at each $\eta$. When $\rho^{e}$ and $\rho_{0}^{e}$ are both calculated to be zero at $\epsilon_{I}$, the solvation free energy $\Delta \mu$ can be obtained without any difficulty through Eqs. (13)-(18). In addition, the evaluation of $\Delta \mu$ can be performed without trouble, as noted in the footnote (55) of Ref. 5, even when $\rho^{e}$ is zero and $\rho_{0}^{e}$ is not. A numerical problem arises when $\rho^{e}$ is nonzero and $\rho_{0}^{e}$ is zero at a particular $\epsilon_{I}$. In this case, $w^{e}$ and $w_{0}^{e}$ at the other energy coordinates can be treated with the procedure given by Eq. (A7) of Ref. 5. Due to Eq. (18), furthermore, $w_{0}^{e}$ at $\epsilon_{I}$ is not needed in the calculation of $\Delta \mu$. The problem is that $w^{e}$ at $\epsilon_{I}$ is required when $\Delta \mu$ is to be evaluated through Eqs. (13)-(18). In the present work, $w^{e}$ at $\epsilon_{I}$ is set equal to the $w^{e}$ at the closest energy coordinate where both of $\rho^{e}$ and $\rho_{0}^{e}$ are nonzero. ${ }^{40,41}$

In the model calculations of Ref. 5, the solute-solvent interaction is restricted to a finite region and the solvent molecules outside the interaction region are excluded in the con- 
struction of the energy distribution functions. In this work, all the solvent molecules are counted in the distribution functions. The solvent molecule interacting weakly with the solute molecule $(\epsilon \approx 0)$ is then large in number, and the discretization of the energy coordinate $\epsilon$ is correspondingly fine near $\epsilon=0$.

${ }^{1}$ J. P. Hansen and I. R. McDonald, Theory of Simple Liquids (Academic, London, 1986).

${ }^{2}$ A. Ben-Naim, Solvation Thermodynamics (Plenum, New York, 1987).

${ }^{3}$ D. Chandler and L. R. Pratt, J. Chem. Phys. 65, 2925 (1976).

${ }^{4}$ N. Matubayasi and M. Nakahara, J. Chem. Phys. 113, 6070 (2000).

${ }^{5}$ N. Matubayasi and M. Nakahara, J. Chem. Phys. 117, 3605 (2002); 118, 2446 (2003)

${ }^{6}$ In Refs. 4 and 5, we employed the terms "chemical potential" and "excess chemical potential" instead of "solvation free energy."

${ }^{7}$ M. P. Allen and D. J. Tildesley, Computer Simulation of Liquids (Oxford University Press, Oxford, 1987).

${ }^{8}$ It is actually sufficient to suppose that only the solute-solvent interaction is pairwise additive.

${ }^{9}$ When the intramolecular degrees of freedom are present in the solvent molecule, the corresponding intramolecular coordinates are contained in $\mathbf{x}$. The overall position and orientation of the solute molecule may also be incorporated into the coordinate $\psi$. Actually, the developments in the present paper are valid simply by defining $\mathbf{x}$ and $\psi$ as collections of the variables that are enough to specify the solute-solvent interaction potential.

${ }^{10}$ According to Eqs. (3) and (6), the solvation free energy $\Delta \mu$ provides the normalization factor for the probability distribution function generated from $p_{0}(\psi) \exp (-\beta \Delta \sigma(\psi))$. Similarly, Eqs. (27) and (28) show that $\Delta \mu$ serves to normalize the probability distribution function proportional to $P_{0}(\phi) \exp (-\beta \Delta \nu(\phi))$.

${ }^{11}$ C. H. Bennett, J. Comput. Phys. 22, 245 (1976).

${ }^{12}$ K. S. Shing and K. E. Gubbins, Mol. Phys. 5, 1109 (1982).

${ }^{13}$ The second term of Eq. (29) is zero only when $P(\phi)=P_{0}(\phi)$ at each $\phi$.

${ }^{14}$ When $\Delta \nu(\phi)$ and $P_{0}(\phi)$ are fixed, the extremization of Eq. (29) with respect to $P(\phi)$ leads to Eq. (28) under the normalization condition for $P(\phi)$.

${ }^{15}$ K. Binder and A. P. Young, Rev. Mod. Phys. 58, 801 (1986).

${ }^{16}$ W. G. Madden, J. Chem. Phys. 96, 5422 (1992).

${ }^{17}$ J. A. Given, Phys. Rev. A 45, 816 (1992).

${ }^{18}$ J. A. Given and G. Stell, J. Chem. Phys. 97, 4573 (1992).

${ }^{19}$ J. A. Given and G. Stell, Physica A 209, 495 (1994).

${ }^{20}$ A. Kovalenko and F. Hirata, J. Chem. Phys. 115, 8620 (2001).

${ }^{21}$ Actually, $\Delta \tilde{\mu}$ evaluated through Eqs. (35)-(39) is exact to second order in the solvent density and in the solute-solvent interaction for any choice of the weight factor $\widetilde{\alpha}(\epsilon)$.

${ }^{22}$ A different choice of $\Phi$ corresponds to a different form of approximation when Eqs. (29) and (35)-(40) are employed.

${ }^{23}$ H. J. C. Berendsen, J. R. Grigera, and T. P. Straatsma, J. Phys. Chem. 91, 6269 (1987).

${ }^{24}$ Of course, a negative value of the distance $r$ is not allowed in Eq. (44). In other words, the intramolecular potential function of the solute molecule is prohibitively large when $r<0$. Actually, the region of $r \leqq 0$ is not available in the practically used form of potential function between two sites. When the two sites are chemically bonded, $K$ in Eq. (44) is much larger the ones employed in the preset work. A nonbonded pair of sites is subject to strong repulsion, typically in the Lennard-Jones form, at short distances. Our values of $K$ are adopted simply to realize substantial fluctuation in the solute structure within the form of Eq. (44).
${ }^{25}$ J. Chandrasekhar, D. C. Spellmeyer, and W. L. Jorgensen, J. Am. Chem. Soc. 106, 903 (1984).

${ }^{26}$ When a molecule carries partial charges and the Ewald method is adopted, its "intramolecular" potential function depends slightly on the overall orientation.

${ }^{27}$ The scheme based on Eq. (6) is effective only when the solute intramolecular coordinate $\psi$ is low-dimensional. When $\psi$ is of high dimension, the calculation of $p(\psi)$ [and possibly $p_{0}(\psi)$ ] is difficult.

${ }^{28}$ P. B. Balbuena, K. P. Johnston, and P. J. Rossky, J. Phys. Chem. 99, 1554 (1995).

${ }^{29}$ P. B. Balbuena, K. P. Johnston, and P. J. Rossky, J. Phys. Chem. 100, 2706 (1996).

${ }^{30}$ R. E. Westacott, K. P. Johnston, and P. J. Rossky, J. Phys. Chem. B 105, 6611 (2001).

${ }^{31}$ In the present calculations of the solvation free energies of the HM-2-1 and HM-5-1 solutes in the original form of approximation, the source of the numerical trouble is the poor statistics of the energy distribution functions $\rho_{0}^{e}(\epsilon)$ and $\chi_{0}^{e}(\epsilon, \eta)$ in the low-energy region of $\epsilon \lessgtr-25 \mathrm{kcal} / \mathrm{mol}$, where the energy coordinate $\epsilon$ was discretized with an interval of $0.1 k_{B} T$, as described in the Appendix of Ref. 5. In principle, when the discretization interval is sufficiently fine and the sampling statistics is good enough, the calculation of the solvation free energy does not involve a numerical trouble even in the original form of approximation. The difficulty in practice is that the important $\epsilon$ region is sampled too rarely when the structure of the solute is largely affected by the solute-solvent interaction.

${ }^{32}$ When $\epsilon \simeq 0, \rho^{e}(\epsilon)$ is observed to be smaller than $\rho_{0}^{e}(\epsilon)$ and $\widetilde{\rho}_{0}^{e}(\epsilon)$. This is actually a finite-size effect of the present calculations. In the limit of large system size, $\rho^{e}(\epsilon) / \rho_{0}^{e}(\epsilon)$ and $\rho^{e}(\epsilon) / \widetilde{\rho}_{0}^{e}(\epsilon)$ need to be unity near $\epsilon=0$.

${ }^{33}$ The number of solvent molecules interacting with the solute varies in response to the change in the system configuration when the solutesolvent interaction is truncated in a finite region. It is not variable, in contrast, when the truncation is not applied and the number of solvent molecules is constant in the ensemble.

${ }^{34}$ The presentation in Appendix B of Ref. 4 is not sufficiently careful at this point. The procedure to fix the additive constant needs to be supplemented to complete the arguments.

${ }^{35} \mathrm{~A}$ similar argument to prove the one-to-one correspondence applies in the full coordinate representation.

${ }^{36} \mathrm{~N}$ fluctuates in the grand canonical ensemble.

${ }^{37}$ When the reference solute molecule is employed, $\int d \epsilon\langle\hat{\rho}(\epsilon)\rangle_{\phi}=N$ holds at each $\phi$ in addition to the counterpart of Eq. $(\mathrm{C} 1)$, where $\langle\cdots)_{\phi}$ is given by Eq. (24).

${ }^{38}$ In the present work, the energy distribution functions obtained from the simulations of the solution and pure solvent systems are stored in seven digits.

${ }^{39} \rho^{e}$ is usually calculated to be zero numerically in the strongly unfavorable region of the solute-solvent interaction even when the corresponding value of the energy coordinate is finite.

${ }^{40}$ The energy coordinate $\epsilon$ is one-dimensional and is discretized in the numerical implementation. In this case, the condition that $\epsilon_{N}>\epsilon$ for any $\epsilon$ satisfying $\rho^{e}(\epsilon)>0$ and $\rho_{0}^{e}(\epsilon)>0$ is fulfilled by only one $\epsilon_{N}$ in the region of $\epsilon<\epsilon_{I}$. In the region of $\epsilon>\epsilon_{I}$, similarly, a unique $\epsilon_{P}$ exists which is smaller than any $\epsilon$ satisfying $\rho^{e}(\epsilon)>0$ and $\rho_{0}^{e}(\epsilon)>0$. $w^{e}\left(\epsilon_{I}\right)$ is then set equal to $w^{e}\left(\epsilon_{N}\right)$ when $\epsilon_{N}$ is closer to $\epsilon_{I}$ than $\epsilon_{P}$, and it is $w^{e}\left(\epsilon_{P}\right)$ when $\epsilon_{P}$ is closer. When $\left(\epsilon_{I}-\epsilon_{N}\right)$ is equal to $\left(\epsilon_{P}-\epsilon_{I}\right), w^{e}\left(\epsilon_{I}\right)$ is simply taken to be $\left(w^{e}\left(\epsilon_{N}\right)+w^{e}\left(\epsilon_{P}\right)\right) / 2$.

${ }^{41}$ When there exist more than one energy coordinates at which $\rho^{e}$ is nonzero and $\rho_{0}^{e}$ is zero, the numerical procedures described can be straightforwardly extended. 